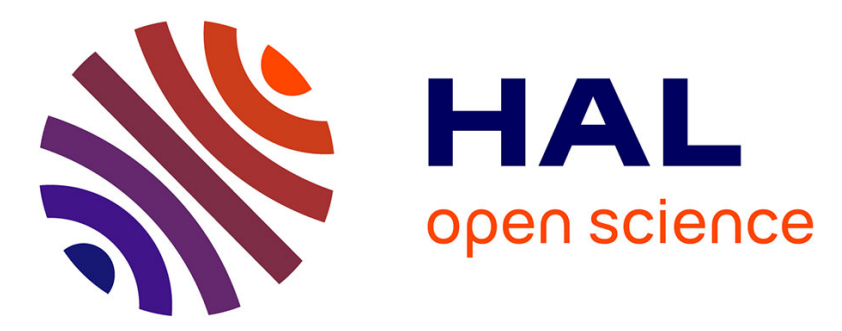

\title{
How to estimate observability constants of one-dimensional wave equations? Propagation versus spectral methods
}

Alain Haraux, Thibault Liard, Yannick Privat

\section{- To cite this version:}

Alain Haraux, Thibault Liard, Yannick Privat. How to estimate observability constants of onedimensional wave equations? Propagation versus spectral methods. Journal of Evolution Equations, 2016, 10.1007/s00028-016-0321-y . hal-01057663v2

\section{HAL Id: hal-01057663 \\ https://hal.science/hal-01057663v2}

Submitted on 7 Apr 2016

HAL is a multi-disciplinary open access archive for the deposit and dissemination of scientific research documents, whether they are published or not. The documents may come from teaching and research institutions in France or abroad, or from public or private research centers.
L'archive ouverte pluridisciplinaire HAL, est destinée au dépôt et à la diffusion de documents scientifiques de niveau recherche, publiés ou non, émanant des établissements d'enseignement et de recherche français ou étrangers, des laboratoires publics ou privés. 


\title{
How to estimate observability constants of one-dimensional wave equations? Propagation versus Spectral methods*
}

\author{
Alain Haraux ${ }^{\dagger, \ddagger}$, Thibault Liard ${ }^{\ddagger}$, , and Yannick Privat ${ }^{\dagger, \ddagger}$ \\ ${ }^{\dagger}$ CNRS, UMR 7598, Laboratoire Jacques-Louis Lions, F-75005, Paris, France. \\ ${ }^{\ddagger}$ Sorbonne Universités, UPMC Univ. Paris 06, UMR 7598, Laboratoire \\ Jacques-Louis Lions, F-75005, Paris, France. \\ (alain.haraux, thibault.liard, yannick.privat@upmc.fr)
}

\begin{abstract}
For a given bounded connected domain in $\mathbb{R}^{n}$, the issue of computing the observability constant associated to a wave operator, an observation time $T$ and a generic observation subdomain constitutes in general a hard task, even for one-dimensional problems. In this work, we introduce and describe two methods to provide precise (and even sharp in some cases) estimates of observability constants for general one dimensional wave equations: the first one uses a spectral decomposition of the solution of the wave equation whereas the second one is based on a propagation argument along the characteristics. Both methods are extensively described and we then comment on the advantages and drawbacks of each one. The discussion is illustrated by several examples and numerical simulations. As a byproduct, we deduce from the main results estimates of the cost of control (resp. the decay rate of the energy) for several controlled (resp. damped) wave equations.
\end{abstract}

Keywords: wave equation, characteristics method, Sturm-Liouville problems, eigenvalues, Ingham's inequality.

AMS classification: 35L05, 93B07, 35Q93, 35B35.

\section{Introduction and main results}

\subsection{Motivations and framework}

In control theory, well-posedness issues often come down to showing that a given observability constant is positive. Nevertheless, from a practical point of view, if the considered observability constant is close to zero, the cost of control may be huge, leading to numerical instability phenomena for instance. For this reason, a precise estimate of the observability constant brings in many cases an interesting and tractable information.

In the context of inverse problems, the observability constant can be interpreted as a quantitative measure of the well-posed character of the problem. In control theory, it is directly related to the cost of control.

When realizing experiments, it may also arise, due to several imprecisions on the operating conditions or on the measures, that one only knows partial informations on the parameters of the

*This work is supported by ANR (AVENTURES - ANR-12-BLAN-BS01-0001-01) 
related inverse/control problem. For instance, one could mention the example of Thermoacoustic tomography where the intensity of measures is often very weak and the physical model is in general simplified before exploiting the measures. In that context, it is interesting to obtain estimates of the observability constant depending only on some parameters of the experiment. In what follows, we concentrate on the observation of the wave equation with a zero-order potential function. As physical parameters, we choose the observability time $T$, possibly the Lebesgue measure of the observation subset and some $L^{\infty}$ bounds on the potential function. In what follows, we will then derive estimates of the observability constant depending only on such parameters.

More precisely, this work is devoted to providing explicit lower bounds of observability constants for one-dimensional wave equations with potential using two different methods and comparing the results. We choose in the sequel potentials that only depend on the space variable since it is often more relevant in view of physical applications. A typical case is the consideration of geophysics waves influenced by the earth rotation. Let us make the frame of our study more precise.

Let $T$ denote a positive constant standing for the observability time. We consider the one dimensional wave equation with potential

$$
\begin{array}{ll}
\partial_{t t} \varphi(t, x)-\partial_{x x} \varphi(t, x)+a(x) \varphi(t, x)=0 & (t, x) \in(0, T) \times(0, \pi), \\
\varphi(t, 0)=\varphi(t, \pi)=0 & t \in[0, T], \\
\varphi(0, x)=\varphi_{0}(x), \partial_{t} \varphi(0, x)=\varphi_{1}(x) & x \in[0, \pi],
\end{array}
$$

where the potential $a(\cdot)$ is a nonnegative function belonging to $L^{\infty}(0, \pi)$. It is well known that for every initial data $\left(\varphi_{0}, \varphi_{1}\right) \in H_{0}^{1}(0, \pi) \times L^{2}(0, \pi)$, there exists a unique solution $\varphi \in$ $C^{0}\left(0, T ; H_{0}^{1}(0, \pi)\right) \cap C^{1}\left(0, T ; L^{2}(0, \pi)\right)$ of the Cauchy problem (1).

Notice that, defining the energy function $E_{\varphi}$ by

$$
\begin{aligned}
E_{\varphi}:[0, T] & \longrightarrow \mathbb{R}_{+} \\
t & \longmapsto \int_{0}^{\pi} \partial_{t} \varphi(t, x)^{2}+\partial_{x} \varphi(t, x)^{2}+a(x) \varphi(t, x)^{2} d x,
\end{aligned}
$$

there holds

$$
E_{\varphi}(0)=E_{\varphi}(t)
$$

for every $t \in[0, T]$ and every solution $\varphi$ of (1).

Let $\omega$ be a given measurable subset of $(0, \pi)$ of positive Lebesgue measure. The equation (1) is said to be observable on $\omega$ in time $T$ if there exists a positive constant $c$ such that,

$$
\int_{0}^{T} \int_{\omega} \partial_{t} \varphi(t, x)^{2} d x d t \geqslant c E_{\varphi}(0),
$$

for all $\left(\varphi_{0}, \varphi_{1}\right) \in H_{0}^{1}(0, \pi) \times L^{2}(0, \pi)$, where $E_{\varphi}(0)=\int_{0}^{\pi}\left(\varphi_{1}(x)^{2}+\varphi_{0}^{\prime}(x)^{2}+a(x) \varphi_{0}(x)^{2}\right) d x$.

Note that in the case where $a(\cdot)=0$, for every subset $\omega$ of $[0, \pi]$ of positive measure, it is well known that the observability inequality (3) is satisfied whenever $T \geqslant 2 \pi$ (see [23]).

If (1) is observable on $\omega$ in time $T$, we denote by $c(T, a, \omega)$ the largest constant in (3), that is

$$
c(T, a, \omega)=\inf _{\substack{\left(\varphi_{0}, \varphi_{1}\right) \in H_{0}^{1}(0, \pi) \times L^{2}(0, \pi) \\\left(\varphi_{0}, \varphi_{1}\right) \neq(0,0)}} \frac{\int_{0}^{T} \int_{\omega} \partial_{t} \varphi(t, x)^{2} d x d t}{\int_{0}^{\pi}\left(\varphi_{1}(x)^{2}+\varphi_{0}^{\prime}(x)^{2}+a(x) \varphi_{0}(x)^{2}\right) d x} .
$$

Even in the simple case $a=0$, it is not obvious to determine the constant $c(T, a, \omega)$ for arbitrary choices of $\omega$. Indeed, a spectral expansion of the solution $\varphi$ on a spectral basis shows the emergence of nontrivial crossed terms. Reformulating this question in terms of the Fourier coefficients of the initial data $\left(\varphi_{0}, \varphi_{1}\right)$, the quantity $c(T, a, \omega)$ is seen as the optimal value of a quadratic functional 
over every sequence $c=\left(c_{j}\right)_{j \in \mathbb{Z}} \in \ell^{2}(\mathbb{C})$ of Fourier coefficients such that $\|c\|_{\ell^{2}(\mathbb{C})}=1$. This leads to a delicate mathematical problem. As it will be highlighted in the sequel, it is quite similar to the well-known open problem of determining what are the best constants in Ingham's inequalities.

This article is devoted to the introduction and description of several methods permiting to determine explicit positive constants $c$ such that the inequality (3) holds for all $\left(\varphi_{0}, \varphi_{1}\right) \in H_{0}^{1}(0, \pi) \times$ $L^{2}(0, \pi)$, or equivalently such that $c(T, a, \omega) \geqslant c$. One also requires that the constant $c$ only depend on $T$, possibly $|\omega|$, as well as some $L^{\infty}$ bounds on the potential function $a(\cdot)$. This way, as mentioned above, it is possible to deal with experiments where one only knows partial informations on the operating conditions.

It is structured as follows: the main results are presented in Section 1.2, as well as the presentation of each method (spectral versus propagation). In Section 3, we present applications of our results to control and stabilization of wave equations. Section 4, is devoted to the illustration of the main results and we provide several numerical simulations to comment on both methods, illustrate and compare them. For the convenience of the reader, most of the proofs are gathered in Section 2.

\subsection{Main results}

In this section, we present the estimates of observability constants obtained using each method. Let $\varphi \in C^{0}\left(0, T ; H_{0}^{1}(0, \pi)\right) \cap C^{1}\left(0, T ; L^{2}(0, \pi)\right)$ denote the solution of (1) with initial data $\varphi(0, \cdot)=$ $\varphi_{0}(\cdot) \in H_{0}^{1}(0, \pi)$ and $\partial_{t} \varphi(0, \cdot)=\varphi_{1}(\cdot) \in L^{2}(0, \pi)$. In the sequel and for the sake of simplicity, the notations $r_{+}$or $r_{-}$will respectively denote $\max _{x \in[0, \pi]} r(x)$ and $\min _{x \in[0, \pi]} r(x)$.

First method: spectral estimates. The first method makes full use of the spectral decomposition

$$
\varphi(t, x)=\sum_{j=1}^{+\infty}\left(a_{j} \cos \left(\lambda_{j} t\right)+b_{j} \sin \left(\lambda_{j} t\right)\right) e_{j}(x)
$$

where $\left\{e_{j}\right\}_{k \in \mathbb{N}^{*}}$ denotes an orthonormal Hilbert basis of $L^{2}(0, \pi)$ consisting of eigenfunctions of the operator $A_{a}=-\partial_{x x}+a(\cdot)$ Id with Dirichlet boundary conditions, associated with the positive eigenvalues $\left(\lambda_{j}^{2}\right)_{j \in \mathbb{N}^{*}}$, and

$$
a_{j}=\int_{0}^{\pi} \varphi_{0}(x) e_{j}(x) d x, \quad b_{j}=\frac{1}{\lambda_{j}} \int_{0}^{\pi} \varphi_{1}(x) e_{j}(x) d x
$$

for every $j \in \mathbb{N}^{*}$.

In the following result, we provide an estimate of the observability constant $c(T, a, \omega)$ that only depends on the parameters $T,|\omega|$ and some $L^{\infty}$ bounds on the potential $a(\cdot)$. It is interesting to note that no assumption is made on the topology of the set $\omega$. However, as highlighted in Remark 3 and in the discussion ending Section 1.2, this approach presents some drawbacks, in particular the fact that it can only be used when the potential function $a(\cdot)$ is close to a constant function.

Theorem 1. Consider a function $a \in L^{\infty}\left(0, \pi ; \mathbb{R}_{+}\right)$writing $a(\cdot)=\bar{a}+r(\cdot)$, where $\bar{a} \in \mathbb{R}$ and $r_{-}>-1$. Let $\omega$ be a measurable subset of $(0, \pi)$ of positive measure and $T(r)=\frac{2 \pi}{\gamma(r)}$ where $\gamma(r)=\frac{3-r_{+}+r_{-}}{\sqrt{4+r_{-}}+\sqrt{1+r_{+}}}$. Assume ${ }^{1}$ that $\|r\|_{\infty}<\alpha_{0}$ where $\alpha_{0}$ denotes the unique ${ }^{2}$ (positive) solution

\footnotetext{
${ }^{1}$ With the notations of this theorem, one has $\|r\|_{\infty}=\max \left\{\left|r_{-}\right|,\left|r_{+}\right|\right\}$.

${ }^{2}$ Uniqueness of $\alpha_{0}$ follows from the fact that the mapping $F: \mathbb{R} \ni \alpha_{0} \mapsto 8\left(e^{\frac{\sqrt{2} \pi \alpha_{0}}{\sqrt{1-\alpha_{0}}}}-1\right)+4 \pi \alpha_{0}$ is increasing.
} 
of the equation

$$
|\omega|-\sin |\omega|=|\omega|\left(8\left(e^{\frac{\sqrt{2} \pi \alpha_{0}}{\sqrt{1-\alpha_{0}}}}-1\right)+4 \pi \alpha_{0}\right) .
$$

Then, (1) is observable on $\omega$ for all $T>T(r)$ with $c(T, a, \omega) \geqslant C_{a}(T,|\omega|)$ where

$$
\begin{gathered}
C_{a}(T,|\omega|)=K_{I}(T, r) \frac{|\omega|-\sin |\omega|-4 \pi\|r\|_{\infty}|\omega|-4 \sqrt{|\omega| D(r)\left(\frac{|\omega|}{2}-\frac{\sin (|\omega|)}{2}-2 \pi\|r\|_{\infty}|\omega|\right)}}{\pi+\left(4 \pi \sqrt{D(r)}+\frac{A(r)}{2}+2 \pi D\right)}, \\
A(r)=\frac{1}{\sqrt{1+r_{-}}}, \quad D(r)=e^{\sqrt{2} \pi\|r\|_{\infty} A(r)}-1, \quad \text { and } \quad K_{I}(T, r)=\frac{2}{\pi}\left(T-\frac{4 \pi^{2}}{\gamma(r)^{2} T}\right) .
\end{gathered}
$$

According to (7), there holds $C_{a}(T,|\omega|)>0$.

Furthermore, in the particular case where $a(\cdot)=0$, one can improve the estimate (8) by setting

$$
C_{0}(T,|\omega|)=\left[\frac{T}{2 \pi}\right](|\omega|-\sin |\omega|)
$$

where the bracket notation stands for the integer floor.

The following remarks are in order.

Remark 1. The assumption $r_{-}>-1$ sufficient when $a \in L^{\infty}\left(0, \pi ; \mathbb{R}_{+}\right)$ensures that the resolvent of the operators $A_{a}$ and $A_{r}$ are compact. Nevertheless, the assumptions of this theorem can be extended to potential functions that are non necessarily nonnegative. More precisely, replacing the assumption $r_{-}>-1$ by $\min \left\{r_{-}, r_{-}+\bar{a}\right\}>-1$ leads to the same conclusion.

Remark 2. Note that the decomposition of the potential as $a(\cdot)=\bar{a}+r(\cdot)$ where $\bar{a} \in \mathbb{R}, r_{-} \leqslant$ $r(\cdot) \leqslant r_{+}$almost everywhere in $(0, \pi)$ with $\min \left\{r_{-}, r_{-}+\bar{a}\right\}>-1$ and $\|r\|_{\infty}<\alpha_{0}$ may be nonunique whenever it holds. As a consequence, it is relevant, at least from a numerical point of view to look for the best decomposition, that is the one maximizing the estimate (8).

Remark 3 (Smallness of $\alpha_{0}$ ). The constant appearing in the statement of Theorem 1 is quite small. Indeed, by using the inequality $e^{h}-1 \geqslant h$ holding for all $h \geqslant 0$, it is easy to obtain

$$
\alpha_{0} \leqslant \frac{1}{(8 \sqrt{2}+4) \pi}\left(1-\frac{\sin (|\omega|)}{|\omega|}\right) .
$$

Numerical computation leads to $\frac{1}{(8 \sqrt{2}+4) \pi}<2.08 \cdot 10^{-2}$ and in particular, $\alpha_{0} \leqslant 2.08 .10^{-2}$.

Remark 4 (Simplifying the condition (7)).

In the statement of Theorem 1, the condition $\|r\|_{\infty}<\alpha_{0}$ is fulfilled as soon as

$$
\|r\|_{\infty} \leqslant \beta_{0}\left(1-\frac{\sin (|\omega|)}{|\omega|}\right)
$$

with $\beta_{0}=1.93 \cdot 10^{-2}$.

The value of $\beta_{0}$ can be easily computed by using the inequality $e^{h}-1 \leqslant h+h^{2}$ whenever $0 \leqslant h \leqslant 1$. This inequality leads to

$$
\frac{1}{\beta_{0}} \leqslant 4 \pi\left(1-\frac{2 \sqrt{2}}{\sqrt{1-\underline{\alpha}}}+\frac{4 \pi \underline{\alpha}}{1-\underline{\alpha}}\right),
$$


where $\underline{\alpha}=\frac{1}{(8 \sqrt{2}+4) \pi}<2.08 .10^{-2}$ and the conclusion follows.

This means that Theorem 1 holds only for very small variations around constant potentials. The next theorem provides an estimate holding for a much larger class of potentials. Nevertheless, as illustrated in Section 4, the interest of the estimate given in Theorem 1 rests upon the fact that it is sharper whenever it can be applied. In particular, we will see that for particular resonant observability times and the choice $a(\cdot)=0$, it coincides with the value of the observability constant $c(T, a, \omega)$.

Remark 5. The constant $K_{I}(T, a)$ introduced in the statement of Theorem 1 is a so-called Ingham constant, first introduced by Ingham in [10]. Ingham's inequality constitutes a fundamental result in the theory of nonharmonic Fourier series. It asserts that, for every real number $\gamma$ and every $T>\frac{2 \pi}{\gamma}$, there exist two positive constants $C_{1}(T, \gamma)$ and $C_{2}(T, \gamma)$ such that for every sequence of real numbers $\left(\mu_{n}\right)_{n \in \mathbb{N}^{*}}$ satisfying

$$
\forall n \in \mathbb{N}^{*} \quad\left|\mu_{n+1}-\mu_{n}\right| \geqslant \gamma
$$

there holds

$$
C_{1}(T, \gamma) \sum_{n \in \mathbb{Z}^{*}}\left|a_{n}\right|^{2} \leqslant \int_{0}^{T}\left|\sum_{n \in \mathbb{Z}^{*}} a_{n} \mathrm{e}^{i \mu_{n} t}\right|^{2} d t \leqslant C_{2}(T, \gamma) \sum_{n \in \mathbb{Z}^{*}}\left|a_{n}\right|^{2},
$$

for every $\left(a_{n}\right)_{n \in \mathbb{N}^{*}} \in \ell^{2}(\mathbb{C})$. Denoting by $C_{1}(T, \gamma)$ and $C_{2}(T, \gamma)$ the optimal constants in (10), several explicit estimates of these constants are provided in [10]. For example, it is proved in this article that

$$
C_{1}(T, \gamma) \geqslant \frac{2}{\pi}\left(T-\frac{4 \pi^{2}}{\gamma^{2} T}\right) \quad \text { and } \quad C_{2}(T, \gamma) \leqslant \frac{20 T}{\min \{2 \pi, \gamma T\}} .
$$

Notice that, up to our knowledge, the best constants in [10] are not known. In the particular case where $\mu_{n}=n$ for every $n \in \mathbb{N}^{*}$, one shows easily that for every $T>2 \pi, C_{1}(T, \gamma)=\left[\frac{T}{2 \pi}\right] \pi$ and $C_{2}(T, \gamma)=C_{1}(T, \gamma)+1$, the bracket notation standing for the integer floor.

In a general way, one could choose $K_{I}(T, r)=C_{1}(T, \gamma(r))$ with $T>\frac{2 \pi}{\gamma(r)}$ in the statement of Theorem 1.

Finally, let us mention that the idea to use Ingham inequalities in control theory is a long story (see for instance $[1,7,11,12,14]$ ).

Remark 6. Notice that, due to our use of Ingham's inequality, the time $T(r)$ needed to apply Theorem 1 is greater than the minimal time of observability (see e.g. [2] for the computation of such a time). This restriction is proper to the use of spectral methods. Indeed, even in the very simple case where the potential $a(\cdot)$ vanishes, the time $T(r)$ is equal to $2 \pi$ and is then greater than the minimal observability time. It should be possible to decrease the time $T(r)$ by using only the asymptotic spectral gap (see e.g. [11]), but our main interest here concerns the observability constant and the methods relying on asymptotic gaps do not usually provide good estimates. Actually, when $\mathrm{T}$ approaches the minimal observabiity time, the observability constant tends to 0 . In particular it is not good to be close to the minimal time when we look for a sharp decay estimate of solutions to the equation with dissipative feedback control.

Particular examples of application of this theorem to observation, control and stabilization of one dimensional wave equations are provided in Section 4.

Second method: a propagation argument. This method makes great use of propagation properties of the wave equation to derive sharp energy estimates and is inspired by [6]. The technique consists in inverting the roles of the time and space variables, and to propagate the 
information from the observation domain to other ones. Although the result presented in the next theorem appears a bit technical, the approach used here is robust and holds for very large choices of potential functions $a(\cdot)$, as it will be commented in Section 4 . Note that a close but non-quantitative result has been obtained in [24, Theorem 4] for semilinear wave equations. In the next result, we keep track of the constants in this method, trying to improve each step by choosing the best possible parameters (see for instance Lemma 5 or Remark 11 in the proof).

In the next result and unlike the framework of Theorem 1, the estimate of the observability constant $c(T, a, \omega)$ only depends on $T,\|a\|_{\infty}$ and the precise knowledge of $\omega$.

Theorem 2. Let $\omega=(\alpha, \beta)$ with $0<\alpha<\beta<\pi$ and $T_{0}=2 \max \{\alpha, \pi-\beta\}$. Define for $\eta>0$ and $k \geqslant 0$, the quantity

$$
K(\eta, \alpha, \beta, k)= \begin{cases}C(\eta)\left(\frac{e^{2 k(\pi-\beta+3 \eta)}-e^{4 k \eta}+e^{-2 k \eta}-e^{-2 k(\alpha+2 \eta)}}{2 k \eta}+1\right) & \text { if } k>0 \\ C(\eta)\left(\frac{\pi-\beta+\alpha}{\eta}+3\right) & \text { if } k=0\end{cases}
$$

where $C(\eta)=\frac{1}{2 \eta}+\max \left\{1, \frac{1}{\eta^{2}}\right\}+\max \left\{1, k^{2}\right\}$. Then, (1) is observable on $\omega$ for all $T>T_{0}$ with $c(T, a, \omega) \geqslant C_{T, T_{0}, a, \lambda}^{\prime}(\alpha, \beta)$ where

- if $T \in\left(T_{0}, 2 T_{0}\right)$, then

$$
\begin{gathered}
C_{T, T_{0}, a, \lambda}^{\prime}(\alpha, \beta)=\max _{(\gamma, \eta) \in \mathcal{A}_{\alpha, \beta, T_{0}, T}}\left(\frac{\gamma}{2}-\sup _{j \in \mathbb{N}^{*}} \frac{\left|\sin \left(\lambda_{j} \gamma\right)\right|}{2 \lambda_{j}}\right) \frac{4\left(T-T_{0}-2 \gamma\right)}{\left(8+T^{2}\right) \gamma K\left(\eta, \alpha, \beta,\|a\|_{\infty}^{1 / 2}\right)} \\
\text { and } \mathcal{A}_{\alpha, \beta, T_{0}, T}=\left\{(\gamma, \eta) \mid \gamma \in\left(0, \frac{T-T_{0}}{4}\right) \text { and } \eta \in\left(0, \min \left\{\frac{T-T_{0}}{16}-\frac{\gamma}{8}, \frac{\beta-\alpha}{4}\right\}\right)\right\},
\end{gathered}
$$

- if $T \geqslant 2 T_{0}$, then

$$
\begin{aligned}
& C_{T, T_{0}, a, \lambda}^{\prime}(\alpha, \beta)=\left[\frac{T}{2 T_{0}}\right] \max _{(\gamma, \eta) \in \mathcal{A}_{\alpha, \beta, T_{0}, T}}\left(\frac{\gamma}{2}-\sup _{j \in \mathbb{N}^{*}} \frac{\left|\sin \left(\lambda_{j} \gamma\right)\right|}{2 \lambda_{j}}\right) \frac{T_{0}-2 \gamma}{\left(2+T_{0}^{2}\right) \gamma K\left(\eta, \alpha, \beta,\|a\|_{\infty}^{1 / 2}\right)} \\
& \text { and } \mathcal{A}_{\alpha, \beta, T_{0}, T}=\left\{(\gamma, \eta) \mid \gamma \in\left(0, \frac{T_{0}}{4}\right) \text { and } \eta \in\left(0, \min \left\{\frac{T_{0}}{16}-\frac{\gamma}{8}, \frac{\beta-\alpha}{4}\right\}\right)\right\} .
\end{aligned}
$$

Remark 7. We stress the fact that, unlike the statement of Theorem 1 , no restriction on the $L^{\infty}$ norm of the function $a(\cdot)$ is needed in the statement of Theorem 2.

Remark 8. The constant $C_{T, a, \lambda}^{\prime}(\alpha, \beta)$ given by (12) or (13) writes as the maximum of a two variables function. Due to the presence of highly nonlinear terms in its expression, the maximum cannot be computed explicitly in general, but is nevertheless easy to compute numerically. It will be illustrated in Section 4.

Remark 9. Notice that, in the case $a(\cdot)=0$, we have $\lambda_{j}=j$ for every $j \in \mathbb{N}^{*}$ and the quantity $\frac{\gamma}{2}-\sup _{j \in \mathbb{N}^{*}} \frac{\left|\sin \left(\lambda_{j} \gamma\right)\right|}{2 \lambda_{j}}$ simplifies to $\frac{\gamma}{2}-\frac{|\sin (\gamma)|}{2}$.

In the general case and to avoid to use the knowledge of the whole spectrum, one can simplify (12) and (13) by noting that

$$
\sup _{j \in \mathbb{N}^{*}} \frac{\left|\sin \left(\lambda_{j} \gamma\right)\right|}{2 \lambda_{j}} \leqslant \frac{\gamma}{2} \sup _{\left\{x \geqslant \lambda_{1} \gamma\right\}} \frac{|\sin x|}{x} .
$$

Remark 10 (Key ingredient of the proof.). The proof of Theorem 2 derives benefit from the propagation properties of Equation (1) along the characteristics. This is illustrated on Figure 1, 
representing the propagation of wavefronts in the time-space domain $(0, \pi) \times(0, T)$ in the case where the observation domain is $\omega=(\alpha, \beta)$. Recall that every point $x_{0} \in(0, \pi)$ generates two characteristics: one is going to the left and the other one to the right, in a symmetrical way. Roughly speaking, the solution $\varphi$ of the wave equation (1) is known on the light-gray rectangle domain, and the propagation properties of the wave equation allow to recover $\varphi$ on the the deepgray domain, provided that the observation time $T$ be large enough.

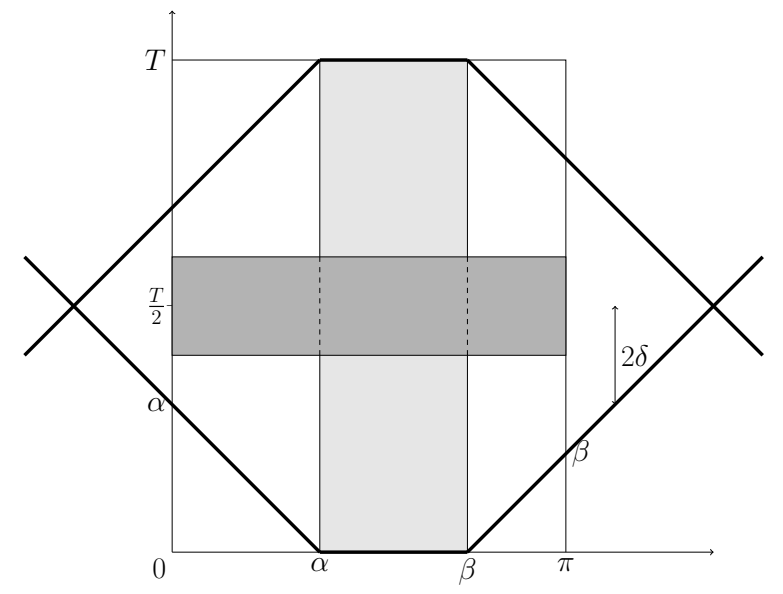

Figure 1: Propagation zones along the characteristics

As a corollary of Theorem 2, we have the following result, extending the estimate of the observability constant to those subsets $\omega$ that are the finite union of open intervals.

Corollary 1. Let $\omega=\bigcup_{i=1}^{n}\left(\alpha_{i}, \beta_{i}\right)$ with $0<\alpha_{1}<\beta_{1}<\cdots<\alpha_{n}<\beta_{n}<\pi$ and $T_{0}=$ $2 \max _{1 \leqslant i \leqslant n}\left\{\alpha_{i}-\beta_{i-1}, \alpha_{i+1}-\beta_{i}\right\}$, with the convention that $\beta_{0}=0$ and $\alpha_{n+1}=\pi$. Define

$$
K^{\prime}\left(\eta_{i}, k\right)= \begin{cases}C\left(\eta_{i}\right)\left(\frac{e^{2 k\left(\alpha_{i+1}-\beta_{i}+3 \eta_{i}\right)}-e^{4 k \eta_{i}}+e^{-2 k \eta_{i}}-e^{-2 k\left(\beta_{i-1}-\alpha_{i}+2 \eta_{i}\right)}}{2 k \eta_{i}}+1\right) & \text { if } k>0 \\ C\left(\eta_{i}\right)\left(\frac{\alpha_{i+1}-\beta_{i}+\alpha_{i}-\beta_{i-1}}{\eta_{i}}+3\right) & \text { if } k=0\end{cases}
$$

for $i \in\{1, \ldots, n\}, \eta_{i}>0$ and $k \geqslant 0$. Then, we have $c(T, a, \omega) \geqslant \min _{1 \leqslant i \leqslant n} C_{T, T_{0}, a, \lambda}^{\prime}\left(\alpha_{i}, \beta_{i}\right)$, where the quantity $K\left(\eta_{i}, \alpha_{i}, \beta_{i}, k\right)$ defined by (11) has been replaced by the quantity $K^{\prime}\left(\eta_{i}, k\right)$ defined by (14) in the definition of the constant $C_{T, T_{0}, a, \lambda}^{\prime}\left(\alpha_{i}, \beta_{i}\right)$.

In Theorem 2 and Corollary 1 , the time $T_{0}$ coincides with the smallest observability time. The following corollary provides a simpler estimate of the observability constant, but is only valid for potentially larger values of the observation time $T$ and provided that we have a precise knowledge of the low frequencies $\left(\lambda_{j}\right)_{j \in \mathbb{N}^{*}}$ associated to the operator $-\partial_{x x}+a(\cdot)$ Id, introduced at the beginning of this section. The proof is based on Ingham inequalities [10].

Corollary 2. Let $\omega=\bigcup_{i=1}^{n}\left(\alpha_{i}, \beta_{i}\right)$ with $0<\alpha_{1}<\beta_{1}<\cdots<\alpha_{n}<\beta_{n}<\pi$. Let $j_{0}=\left[\frac{\|a\|_{\infty}-1}{2}\right]+1$ and introduce the positive real numbers

$$
T_{0}^{\prime}=\frac{2 \pi}{\gamma_{j_{0}}} \quad \text { and } \quad \gamma_{j_{0}}=\left\{\begin{array}{ll}
\frac{1+2 j_{0}-\|a\|_{\infty}}{j_{0}+1+\sqrt{j_{0}^{2}+\|a\|_{\infty}}} & \text { if } j_{0}=1 \\
\min \left\{\min _{j \in\left\{1 \ldots j_{0}-1\right\}} \lambda_{j+1}-\lambda_{j}, \frac{1+2 j_{0}-\|a\|_{\infty}}{j_{0}+1+\sqrt{j_{0}^{2}+\|a\|_{\infty}}}\right\} & \text { if } j_{0}>1
\end{array} .\right.
$$


Then, we have

$$
c(T, a, \omega) \geqslant \widetilde{C}_{T, T_{0}^{\prime}, a, \lambda}(\omega)=\frac{\left(T-T_{0}^{\prime}\right)\left(\gamma_{j_{0}}^{2} T^{2}-4 \pi^{2}\right) \min \left\{2 \pi, \gamma_{j_{0}}, T\right\}}{2\left(\gamma_{j_{0}}^{2} T^{2}-4 \pi^{2}\right) \min \left\{2 \pi, \gamma_{j_{0}}, T\right\}+20 \pi \gamma_{j_{0}}^{2} T^{2}} \Lambda_{T, T_{0}^{\prime}, a}(\omega)
$$

with

$$
\Lambda_{T, T_{0}^{\prime}, a}(\omega)=\min _{1 \leqslant i \leqslant n} \max \left\{\frac{1}{K\left(\eta, \alpha_{i}, \beta_{i},\|a\|_{\infty}^{1 / 2}\right)}, 0<\eta<\min \left\{\frac{T-T_{0}^{\prime}}{16}, \frac{\beta_{i}-\alpha_{i}}{4}\right\}\right\} .
$$

Short discussion on the main results. We stress the fact that the main ingredients in the proofs of Theorem 1 on the first hand, and Theorem 2, Corollaries 1 and 2 are of different natures. Indeed, Theorem 1 is based on a purely spectral argument, and is somehow limited by the misreading of the low frequencies of the spectrum. This explains the restrictions on the norm of the potential $\|a\|_{\infty}$. By the way, notice that the knowledge of the value of a spectral gap $\gamma$ (in the sense made precise in Remark 5) would permit to avoid the technicalities in Lemmas 2, 3 and 4 and to get directly a simple estimate instead of the one of Theorem 2. The results in Theorem 2, Corollaries 1 and 2 are in some sense more robust since we do not need to make assumptions on the smallness of the difference between the maximal and minimal values of the potential $a(\cdot)$. Moreover, on the contrary to the spectral method, propagation ones provide a lower bound of the observability constant working when the observation time $T$ is close to the minimal observation time $T_{0}$ when $\omega$ is an interval.

Recall also that, independently of the aforementioned drawbacks, in the context of an inverse problem where there is some indetermination on some parameters of the problem, Theorem 1 can be used when the topological nature (in particular the number of connected components) of $\omega$ is not known whereas Theorem 2 assumes that $\omega$ is known and writes as a finite union of intervals. In each case, one can assume that only $L^{\infty}$ bounds are known on the potential $a(\cdot)$.

To sum-up, we gather this discussion under the following condensed form.

\begin{tabular}{|c|c|c|c|}
\hline & requires the knowledge of & Advantage & Drawback \\
\hline \hline $\begin{array}{c}\text { Spectral } \\
\text { method }\end{array}$ & $T,|\omega|, L^{\infty}$ bounds on $a(\cdot)$ & $\begin{array}{l}\text { - sharp estimate, } \\
-\omega \text { is only assumed to } \\
\text { be measurable }\end{array}$ & $\begin{array}{c}\text { works for almost } \\
\text { constant potentials }\end{array}$ \\
\hline $\begin{array}{c}\text { Propagation } \\
\text { method }\end{array}$ & $T, \omega, L^{\infty}$ bounds on $a(\cdot)$ & $\begin{array}{c}\text { no restriction on the } \\
\text { potentials }\end{array}$ & $\begin{array}{l}\text { - estimate not so accu- } \\
\text { rate } \\
-\omega \text { writes as a finite } \\
\text { union of intervals }\end{array}$ \\
\hline
\end{tabular}

Section 4 is devoted to the numerical comparison and results obtained using each method.

\section{Proofs of the main results}

\subsection{Proof of Theorem 1}

Let us begin by proving the second part of the statement of Theorem 1, the general case using strongly the estimate obtained in this simple case.

The case $a(\cdot)=0$. Assume that $a(\cdot)=0$. According to (5) and since the eigenfunctions of the Dirichlet-Laplacian operator on $\Omega=(0, \pi)$ are given by $e_{j}(x)=\sqrt{\frac{2}{\pi}} \sin (j x)$ for every $j \in \mathbb{N}^{*}$ it 
follows that for all initial data $\left(\varphi^{0}, \varphi^{1}\right) \in H_{0}^{1}(0, \pi) \times L^{2}(0, \pi)$, the solution $\varphi \in C^{0}\left(0, T ; H_{0}^{1}(0, \pi)\right) \cap$ $C^{1}\left(0, T ; L^{2}(0, \pi)\right)$ of $(1)$ can be expanded as

$$
\varphi(t, x)=\sum_{j=1}^{+\infty}\left(a_{j} \cos (j t)+b_{j} \sin (j t)\right) \sin (j x),
$$

where the sequences $\left(j a_{j}\right)_{j \in \mathbb{N}^{*}}$ and $\left(j b_{j}\right)_{j \in \mathbb{N}^{*}}$ belong to $\ell^{2}(\mathbb{R})$ and are determined in function of the initial data $\left(\varphi^{0}, \varphi^{1}\right)$ by

$$
a_{j}=\frac{2}{\pi} \int_{0}^{\pi} \varphi^{0}(x) \sin (j x) d x, \quad b_{j}=\frac{2}{j \pi} \int_{0}^{\pi} \varphi^{1}(x) \sin (j x) d x,
$$

for every $j \in \mathbb{N}^{*}$.

By the way, note that

$$
\left\|\left(\varphi^{0}, \varphi^{1}\right)\right\|_{H_{0}^{1} \times L^{2}}^{2}=\frac{\pi}{2} \sum_{j=1}^{+\infty} j^{2}\left(a_{j}^{2}+b_{j}^{2}\right),
$$

and furthermore, one has

$$
\begin{aligned}
\int_{0}^{T} \int_{\omega}\left|\partial_{t} \varphi(t, x)\right|^{2} d x d t & \geqslant \int_{\omega} \int_{0}^{2 \pi\left[\frac{T}{2 \pi}\right]}\left|\partial_{t} \varphi(t, x)\right|^{2} d t d x \\
& =\left[\frac{T}{2 \pi}\right] \int_{\omega} \int_{0}^{2 \pi}\left|\sum_{j=1}^{+\infty} j\left(-a_{j} \sin (j t)+b_{j} \cos (j t)\right) \sin (j x)\right|^{2} d t d x \\
& =\pi\left[\frac{T}{2 \pi}\right] \sum_{j=1}^{\infty} j^{2}\left(a_{j}^{2}+b_{j}^{2}\right) \int_{\omega} \sin ^{2}(j x) d x
\end{aligned}
$$

The following lemma is a crucial ingredient to conclude.

Lemma 1. Let $j \in \mathbb{N}^{*}$. For every measurable subset $\omega$ of $(0, \pi)$, one has

$$
\int_{\omega} \sin (j x)^{2} d x \geqslant \frac{|\omega|-\sin |\omega|}{2} .
$$

This lemma is noticed as well in [18, 20] and used for controllability purposes in [15]. Even though it is well known, we provide at the end of this paragraph an elementary and new proof of this result using the Schwarz symmetrization.

As a consequence, one gets

$$
\int_{0}^{T} \int_{\omega}\left|\partial_{t} \varphi(t, x)\right|^{2} d x d t \geqslant \frac{\pi}{2}\left[\frac{T}{2 \pi}\right](|\omega|-\sin |\omega|) \sum_{j=1}^{\infty} j^{2}\left(a_{j}^{2}+b_{j}^{2}\right) .
$$

Combining this inequality with (18), we infer

$$
\left[\frac{T}{2 \pi}\right](|\omega|-\sin |\omega|) \int_{0}^{\pi}\left(\varphi_{1}(x)^{2}+\varphi_{0}^{\prime}(x)^{2}\right) d x \leqslant \int_{0}^{T} \int_{\omega} \partial_{t} \varphi(t, x)^{2} d x d t
$$

whence the estimate (9). 
Proof of Lemma 1. Let us first estimate the quantity

$$
\delta_{j}(L)=\sup \left\{\int_{\omega} f_{j}(x) d x, \omega \text { measurable subset of }(0, \pi) \text { such that }|\omega|=L \pi\right\},
$$

where $f_{j}(x)=\sin (j x)^{2}$ and $L \in(0,1)$ is fixed. Let $\omega$ be a measurable subset of $(0, \pi)$ such that $|\omega|=L \pi$. Denote by $f_{j, S}$ the Schwarz rearrangement ${ }^{3}$ of the function $f_{j}$. According to the Hardy-Littlewood inequality (see e.g. [9, 13]), one has

$$
\int_{\omega} f_{j}(x) d x=\int_{0}^{\pi} \chi_{\omega}(x) f_{j}(x) d x \leqslant \int_{0}^{\pi} \chi_{\omega_{S}}(x) f_{j, S}(x) d x=\int_{\omega_{S}} f_{j, S}(x) d x .
$$

Moreover, notice that $f_{j, S}=f_{1}$ for every $j \in \mathbb{N}^{*}$. Indeed, introducing for every $\mu \in(0,1)$ and $j \in \mathbb{N}^{*}$ the set $\Omega_{j}(\mu)=\left\{x \in(0, \pi) \mid f_{j}(x) \geqslant \mu\right\}$, a simple computation ensures that

$$
\Omega_{j}(\mu)=\bigcup_{k=1}^{j}\left\{x \in(0, \pi)|| x-\frac{(2 k-1) \pi}{2 j} \mid \leqslant \frac{(2 k-1) \pi}{j}-\frac{\arcsin (\sqrt{\mu})}{j}\right\},
$$

so that, $\left|\Omega_{j}(\mu)\right|=\pi-2 \arcsin (\sqrt{\mu})=\left|\Omega_{1}(\mu)\right|$. The expected result follows easily.

As a consequence and since $\omega_{S}=((1-L) \pi / 2,(1+L) \pi / 2)$, one has

$$
\int_{\omega} f_{j}(x) d x \leqslant \int_{\omega_{S}} f_{1}(x) d x=\frac{L \pi+\sin (L \pi)}{2} .
$$

and then $\delta_{j}(L) \leqslant \frac{L \pi+\sin (L \pi)}{2}$ for every $j \in \mathbb{N}^{*}$. Finally, denoting by $\omega^{c}$ the complement set of $\omega$ in $(0, \pi)$, we get the expected inequality by writing

$$
\begin{aligned}
\inf _{\substack{\omega \\
|\omega|=L \text { masable }}} \int_{\omega} \sin (j x)^{2} d x & =\frac{\pi}{2}-\sup _{\substack{\omega^{c} \text { measurable } \\
\left|\omega^{c}\right|=(1-L) \pi}} \int_{\omega^{c}} \sin (j x)^{2} d x \\
& \geqslant \frac{\pi}{2}-\left(\frac{(1-L) \pi+\sin ((1-L) \pi)}{2}\right)=\frac{L \pi-\sin (L \pi)}{2} .
\end{aligned}
$$

We thus infer that

$$
\int_{\omega} \sin (j x)^{2} d x \geqslant \frac{|\omega|-\sin |\omega|}{2}
$$

for every measurable subset $\omega$ of $(0, \pi)$.

The case $\bar{a}=0$. We start with some elementary lemmas that play an important role.

Lemma 2. Assume that $r_{-}>-1$ and $r_{+}-r_{-}<3$. Then, for every $j \in \mathbb{N}^{*}$, one has

$$
\lambda_{j+1}-\lambda_{j} \geqslant \frac{3-r_{+}+r_{-}}{\sqrt{4+r_{-}}+\sqrt{1+r_{+}}} .
$$

\footnotetext{
${ }^{3}$ For every subset $U$ of $\Omega$, we denote by $U_{S}$ the ball centered at $\frac{\pi}{2}$ having the same Lebesgue measure as $U$. We recall that, for every nonnegative Lebesgue measurable function $u$ defined on $\Omega$ and vanishing on its boundary, denoting by $\Omega(c)=\{x \in \Omega \mid u(x) \geqslant c\}$ its level sets, the Schwarz rearrangement of $u$ is the function $u_{S}$ defined on $\Omega_{S}$ by

$$
u_{S}(x)=\sup \left\{c \mid x \in(\Omega(c))_{S}\right\} .
$$

The function $u_{S}$ is built from $u$ by rearranging the level sets of $u$ into balls having the same Lebesgue measure (see, e.g., [9, Chapter 2]).
} 
Proof. The Courant-Fischer minimax principle writes

$$
\lambda_{j}^{2}=\min _{\substack{V \subset H_{0}^{1}(0, \pi) \\ \operatorname{dim} V=j}} \max _{u \in V \backslash\{0\}} \frac{\int_{0}^{\pi}\left(u^{\prime}(x)^{2}+r(x) u(x)^{2}\right) d x}{\int_{0}^{\pi} u(x)^{2} d x} .
$$

Using that $r_{-} \leqslant r(x) \leqslant r_{+}$for almost every $x \in(0, \pi)$ yields

$$
\sqrt{j^{2}+r_{-}} \leqslant \lambda_{j} \leqslant \sqrt{j^{2}+r_{+}},
$$

for every $j \in \mathbb{N}^{*}$. It suffices indeed to compare $\lambda_{j}^{2}$ with the $j$-th eigenvalue of a Sturm-Liouville operator with constant coefficients. We infer

$$
\begin{aligned}
\lambda_{j+1}-\lambda_{j} & \geqslant \sqrt{(j+1)^{2}+r_{-}}-\sqrt{j^{2}+r_{+}}, \\
& =\frac{1+2 j-r_{+}+r_{-}}{\sqrt{(j+1)^{2}+r_{-}}+\sqrt{j^{2}+r_{+}}},
\end{aligned}
$$

for every $j \in \mathbb{N}^{*}$. The sequence $j \mapsto \sqrt{(j+1)^{2}+r_{-}}-\sqrt{j^{2}+r_{+}}$being increasing, the expected estimate follows.

Notice that similar computations on the asymptotic spectral gap of Sturm-Liouville operators may be found in [19].

Lemma 3. Let $j \in \mathbb{N}^{*}, r \in L^{\infty}(0, \pi)$ such that $r_{-}>-1$ and $\omega$ be a measurable subset of $(0, \pi)$. The $j$-th eigenfunction $e_{j}(\cdot)$ of the operator $A_{r}=-\partial_{x x}+r(\cdot)$ Id satisfies

$$
e_{j}(x)^{2} \geqslant \frac{2}{C_{j}}\left(\sin \left(\lambda_{j} x\right)^{2}+2 \lambda_{j} h_{j}(x) \sin \left(\lambda_{j} x\right)\right)
$$

for every $x \in[0, \pi]$, where

$$
C_{j}=\pi+\frac{2}{\lambda_{j}}\left(2 \pi \sqrt{\left(1+r_{-}\right)\left(e^{\tau(r) \pi}-1\right)}+\frac{1}{4}+\frac{\pi\left(1+r_{-}\right)\left(e^{\tau(r) \pi}-1\right)}{\lambda_{j}}\right),
$$

$h_{j} \in L^{\infty}(0, \pi)$ is such that $\left\|h_{j}\right\|_{\infty}^{2} \leqslant \frac{\left(1+r_{-}\right)\left(e^{\tau(r) \pi}-1\right)}{\lambda_{j}^{4}}$ and $\tau(r)=\frac{\sqrt{2}\|r\|_{\infty}}{\sqrt{1+r_{-}}}$.

Proof. The estimate (20) is obtained by using a shooting method that we roughly describe. Introduce $\psi_{j}: x \mapsto \frac{\sin \left(\lambda_{j} x\right)}{\lambda_{j}}$. The function $\phi_{j}=\frac{e_{j}(\cdot)}{e_{j}^{\prime}(0)}$ solves the following Cauchy system

$$
\begin{aligned}
& -\phi_{j}^{\prime \prime}(x)+r(x) \phi_{j}(x)=\lambda_{j}^{2} \phi_{j}(x) \quad x \in(0, \pi), \\
& \phi_{j}(0)=0, \quad \phi_{j}^{\prime}(0)=1,
\end{aligned}
$$

whereas the function $h_{j}=\phi_{j}-\psi_{j}$ solves the Cauchy system

$$
\begin{aligned}
& -h_{j}^{\prime \prime}(x)+\left(r(x)-\lambda_{j}^{2}\right) h_{j}(x)=-r(x) \psi_{j}(x) \quad x \in(0, \pi), \\
& h_{j}(0)=0, \quad h_{j}^{\prime}(0)=0 .
\end{aligned}
$$

Let us denote by $\mathcal{V}_{\lambda_{j}}$ the energy function defined by

$$
\mathcal{V}_{\lambda_{j}}(x)=\frac{1}{2} h_{j}^{\prime 2}(x)+\frac{\lambda_{j}^{2}}{2} h_{j}^{2}(x) .
$$


Using (22), one gets the estimate

$$
\mathcal{V}_{\lambda_{j}}^{\prime}(x) \leqslant \tau(r) \mathcal{V}_{\lambda_{j}}(x)+\frac{\|r\|_{\infty}^{2}}{\tau(r) \lambda_{j}^{2}}
$$

for every $x \in[0, \pi]$, with $\tau(r)=\frac{\sqrt{2}\|r\|_{\infty}}{\sqrt{1+r_{-}}}$. According to the Gronwall lemma, we infer

$$
\left\|h_{j}\right\|_{\infty}^{2} \leqslant \frac{1+r_{-}}{\lambda_{j}^{4}}\left(e^{\tau(r) \pi}-1\right) .
$$

As a consequence, and since $e_{j}(\cdot)=\frac{\phi_{j}(\cdot)}{\sqrt{\int_{0}^{\pi} \phi_{j}(x)^{2} d x}}$, one gets

$$
\int_{0}^{\pi} \phi_{j}(x)^{2} d x=\int_{0}^{\pi}\left(\frac{\sin ^{2}\left(\lambda_{j} x\right)}{\lambda_{j}^{2}}+2 h_{j}(x) \frac{\sin \left(\lambda_{j} x\right)}{\lambda_{j}}+h_{j}(x)^{2}\right) d x \leqslant \frac{C_{j}}{2 \lambda_{j}^{2}},
$$

where $C_{j}$ is defined by (21). The expected estimate then follows.

Lemma 4. Let $r \in L^{\infty}(0, \pi)$ such that $r_{-}>-1$. Then, one has

$$
\int_{\omega} \sin ^{2}\left(\lambda_{j} x\right) d x \geqslant \frac{|\omega|}{2}-\frac{\sin |\omega|}{2}-\frac{2 \pi\|r\|_{\infty}|\omega|}{j},
$$

for every $j \in \mathbb{N}^{*}$.

Proof. In accordance with (19), let us write $\lambda_{j}=j+\frac{\ell_{j}}{j}$ with $r_{-} \leqslant l_{j} \leqslant r_{+}$for every $j \in \mathbb{N}^{*}$. Using Lemma 1, we get

$$
\begin{aligned}
\int_{\omega} \sin ^{2}\left(\lambda_{j} x\right) d x & =\frac{|\omega|}{2}-\frac{1}{2} \int_{\omega} \cos \left(2 \lambda_{j} x\right) d x \\
& =\int_{\omega}\left(\sin ^{2}(j x)+\frac{\ell_{j}}{j} \cos (2 j x) \int_{0}^{x} \sin \left(\frac{2 s \ell_{j}}{j}\right) d s+\frac{1}{2} \sin (2 j x) \sin \left(\frac{2 \ell_{j} x}{j}\right)\right) d x \\
& \geqslant \frac{|\omega|}{2}-\frac{\sin |\omega|}{2}-\frac{2 \pi\left|l_{j}\right||\omega|}{j} .
\end{aligned}
$$

Since $\left|l_{j}\right| \leqslant\|r\|_{\infty}$

$$
\int_{\omega} \sin ^{2}\left(\lambda_{j} x\right) d x \geqslant \frac{|\omega|}{2}-\frac{\sin |\omega|}{2}-\frac{2 \pi\|r\|_{\infty}|\omega|}{j}
$$

We now prove Theorem 1. Combining (20) with the Cauchy-Schwarz inequality, one gets

$$
\int_{\omega} e_{j}(x)^{2} d x \geqslant \frac{2}{C_{j}}\left(\int_{\omega} \sin ^{2}\left(\lambda_{j} x\right) d x-2 \lambda_{j} \sqrt{\int_{\omega} h_{j}(x)^{2} d x} \sqrt{\int_{\omega} \sin ^{2}\left(\lambda_{j} x\right) d x}\right)
$$

for every $j \in \mathbb{N}^{*}$. The positivity of the right hand side in the inequality above is equivalent to the condition $\int_{\omega} \sin ^{2}\left(\lambda_{j} x\right) d x>4 \lambda_{j}^{2} \int_{\omega} h_{j}(x)^{2} d x$. Moreover, according to Lemma 3, one shows easily that this condition holds whenever $\|r\|_{\infty}<\alpha_{0}$, where $\alpha_{0}$ denotes the unique solution of (7) with 
$\bar{a}=0$. Finally, the constant $C_{j}$ being defined by (21), one finally gets using the estimate (19) an upper bound on it, uniform with respect to $j$, so that

$$
\int_{\omega} e_{j}(x)^{2} d x \geqslant \frac{|\omega|-\sin |\omega|-4 \pi\|r\|_{\infty}|\omega|-4 \sqrt{|\omega| D(r)\left(\frac{|\omega|}{2}-\frac{\sin (|\omega|)}{2}-2 \pi\|r\|_{\infty}|\omega|\right)}}{\pi+\left(4 \pi \sqrt{D(r)}+\frac{A(r)}{2}+2 \pi D(r)\right)},
$$

for every $j \in \mathbb{N}^{*}$, using the notations introduced in the statement of Theorem 1 .

By decomposing the solution $\varphi$ of (1) in the spectral basis $\left\{e_{j}\right\}_{j \in \mathbb{N}^{*}}$ as in (5)-(6), one gets using the so-called Ingham inequality

$$
\begin{aligned}
\int_{0}^{T} \int_{\omega}\left|\partial_{t} \varphi(t, x)\right|^{2} d x d t & =\frac{1}{2} \int_{0}^{T} \int_{\omega}\left|\sum_{k \in \mathbb{Z}^{*}} i \operatorname{sgn}(k) \lambda_{|k|} \sqrt{a_{|k|}^{2}+b_{|k|}^{2}} e^{i \operatorname{sgn}(k)\left(\lambda_{|k|} t-\theta_{|k|}\right)} e_{|k|}(x)\right|^{2} d t d x \\
& \geqslant \frac{K_{I}(T, a)}{2} \sum_{k \in \mathbb{Z}^{*}}\left(a_{|k|}^{2}+b_{|k|}^{2}\right) \lambda_{|k|}^{2} \int_{\omega} e_{|k|}(x)^{2} d x \\
& =K_{I}(T, a) \sum_{j=1}^{+\infty}\left(a_{j}^{2}+b_{j}^{2}\right) \lambda_{j}^{2} \int_{\omega} e_{j}(x)^{2} d x
\end{aligned}
$$

where $\left(\theta_{j}\right)_{j \in \mathbb{N}^{*}}$ denotes the sequence defined by $e^{i \theta_{j}}=\frac{a_{j}+i b_{j}}{\sqrt{a_{j}^{2}+b_{j}^{2}}}$ for every $j \in \mathbb{N}^{*}$, and $K_{I}(T, a)$ is the Ingham constant introduced in the statement of Theorem 1 and whose choice is commented in Remark 5.

In the sequel and for the sake of simplicity, the notations $\varphi_{x}$ or $\varphi_{t}$ will respectively denote the partial derivatives $\partial_{x} \varphi$ and $\partial_{t} \varphi$.

The energy identity

$$
\int_{0}^{\pi}\left(\varphi_{t}^{2}(t, x)+\varphi_{x}^{2}(t, x)+a(x) \varphi^{2}(t, x)\right) d x=\sum_{j=1}^{+\infty} \lambda_{j}^{2}\left(a_{j}^{2}+b_{j}^{2}\right),
$$

holding for almost every $t \in[0, T]$, we infer

$$
\int_{0}^{T} \int_{\omega}\left|\partial_{t} \varphi(t, x)\right|^{2} d x d t \geqslant K_{I}(T, a) \inf _{j \in \mathbb{N}^{*}} \int_{\omega} e_{j}(x)^{2} d x \int_{0}^{\pi}\left(\varphi_{t}^{2}(t, x)+\varphi_{x}^{2}(t, x)+a(x) \varphi^{2}(t, x)\right) d x .
$$

Finally, the combination of this inequality with (23) provides the desired result. Furthermore, by construction, the right-hand side of the obtained inequality, denoted $C_{a}(T,|\omega|)$ is positive.

The general case. The general estimates are obtained by mimicking the proof in the case where $\bar{a}=0$. Indeed, note that $\lambda_{j}^{2}$ is an eigenvalue of the operator $A_{a}$ if, and only if $\mu_{j}^{2}=\lambda_{j}^{2}-\bar{a}$ is an eigenvalue of the operator $A_{r}$. Since $\mu_{j}^{2} \geqslant \sqrt{1+r_{-}}>0$, one has

$$
\mu_{j+1}-\mu_{j}=\frac{\lambda_{j+1}^{2}-\lambda_{j}^{2}}{\sqrt{\lambda_{j+1}^{2}-\bar{a}}+\sqrt{\lambda_{j}^{2}-\bar{a}}} \geqslant \frac{3-\left(r_{+}-r_{-}\right)}{\sqrt{4+r_{-}}+\sqrt{1+r_{+}}}
$$

for every $j \in \mathbb{N}^{*}$. We obtain successively the same estimates as those in the statements of Lemmas 3 and 4 , replacing the quantity $\lambda_{j}$ by $\mu_{j}$. The expected conclusion follows. 


\subsection{Proof of Theorem 2}

Let us denote by $\varphi$ the unique solution of the wave equation (1) with a given potential $a(\cdot) \in$ $L^{\infty}(0, \pi)$ and with initial data $\left(\varphi(0, \cdot), \partial_{t} \varphi(0, \cdot)\right)=\left(\varphi_{0}, \varphi_{1}\right) \in H_{0}^{1}(0, \pi) \times L^{2}(0, \pi)$.

Using the notations introduced in Section 1, let us define the function $E_{a}$ by

$$
\begin{aligned}
E_{a}:[0, T] \times[0, \pi] & \longrightarrow \mathbb{R}_{+} \\
(t, x) & \longmapsto \partial_{t} \varphi(t, x)^{2}+\partial_{x} \varphi(t, x)^{2}+a(x) \varphi(t, x)^{2},
\end{aligned}
$$

and the function $E_{k^{2}}$ by

$$
\begin{aligned}
E_{k^{2}}:[0, T] \times[0, \pi] & \longrightarrow \mathbb{R}_{+} \\
(t, x) & \longmapsto \partial_{t} \varphi(t, x)^{2}+\partial_{x} \varphi(t, x)^{2}+k^{2} \varphi(t, x)^{2},
\end{aligned}
$$

where $k^{2}=\|a\|_{\infty}$.

This proof is divided into several steps and is illustrated on Figure 2. Let us comment on this figure: in a nutshell, the main ingredients of the proof are contained in the statements of Lemmas 6 and 7, that take advantage of the propagation properties of the wave equation (1) to derive energy estimates on the zone $P_{1}$ (respectively $P_{2}$ ) from the observation on the zone $Q_{1}$ (respectively $Q_{2}$ ). Finally, since we will establish estimates along the characteristics, the space and time variables will play symmetric roles in the algebraic computations that follows.

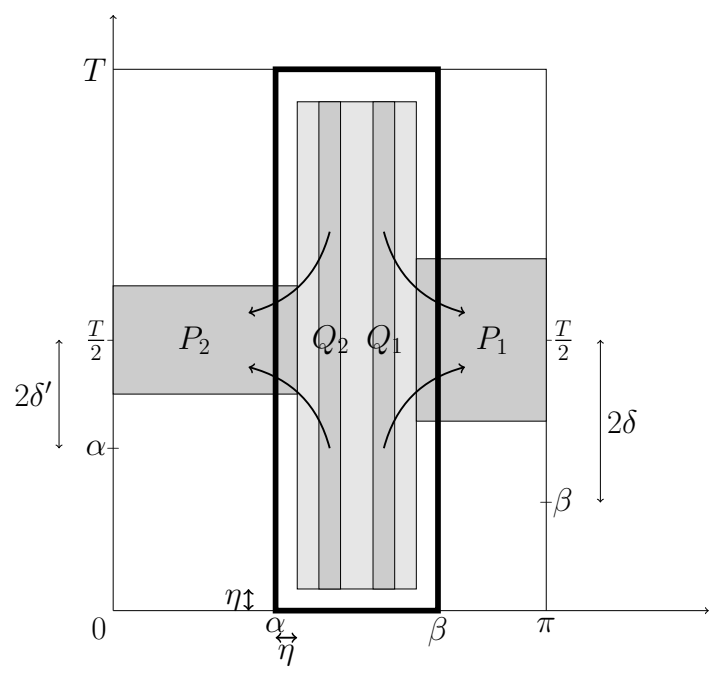

Figure 2: Scheme of the proof (Propagation method)

Let us start with several instrumental lemmas.

The first one states an observability result in the case where $\omega=\Omega=(0, \pi)$.

Lemma 5. Let $\gamma \in(0, T / 2)$. Thus,

$$
\inf _{\left(\varphi_{0}, \varphi_{1}\right) \in H_{0}^{1}(0, \pi) \times L^{2}(0, \pi)} \frac{\int_{\frac{T}{2}-\gamma}^{\frac{T}{2}} \int_{\Omega}\left|\partial_{t} \varphi(t, x)\right|^{2} d x d t}{\int_{\Omega}\left(\varphi_{1}(x)^{2}+\varphi_{0}^{\prime}(x)^{2}+a(x) \varphi_{0}(x)^{2}\right) d x}=\frac{\gamma}{2}-\sup _{j \in \mathbb{N}^{*}} \frac{\left|\sin \left(\lambda_{j} \gamma\right)\right|}{2 \lambda_{j}},
$$

where $\varphi$ denotes the unique solution of the wave equation (1) with initial data $\left(\varphi(0, \cdot), \partial_{t} \varphi(0, \cdot)\right)=$ $\left(\varphi_{0}, \varphi_{1}\right) \in H_{0}^{1}(0, \pi) \times L^{2}(0, \pi)$ and $\left(\lambda_{j}\right)_{j \in \mathbb{N}^{*}}$ the sequence of eigenvalues introduced in Section 1.2. 
Proof. Denote by LHS the left hand side in (24). Decomposing $\varphi$ as in (5)-(6) yields

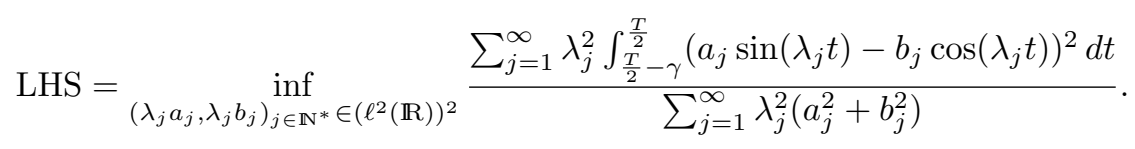

Next, setting $\lambda_{j} a_{j}=\rho_{j} \cos \left(\theta_{j}\right), \lambda_{j} b_{j}=\rho_{j} \sin \left(\theta_{j}\right)$ with $\left(\rho_{j}\right)_{j \in \mathbb{N}^{*}} \in \ell^{2}(\mathbb{R})$ and $\theta_{j} \in \mathbb{T}$ for all $j \in \mathbb{N}^{*}$, we get using a homogeneity argument

$$
\begin{aligned}
\text { LHS } & =\inf _{\sum_{j=1}^{\infty} \rho_{j}^{2}=1} \inf _{\left(\theta_{j}\right)_{j \in \mathbb{N}^{*}}} \sum_{j=1}^{\infty} \rho_{j}^{2} \int_{\frac{T}{2}-\gamma}^{\frac{T}{2}} \sin ^{2}\left(\lambda_{j} t-\theta_{j}\right) d t, \\
& =\frac{\gamma}{2}-\sup _{\sum_{j=1}^{\infty} \rho_{j}^{2}=1} \sup _{\left(\theta_{j}\right)_{j \in \mathbb{N}^{*}}} \sum_{j=1}^{+\infty} \rho_{j}^{2} \frac{\sin \left(\lambda_{j} \gamma\right)}{2 \lambda_{j}} \cos \left(\lambda_{j}(T-\gamma)-2 \theta_{j}\right) .
\end{aligned}
$$

To reach the maximum, it suffices to choose $\theta_{j}$ such that $\cos \left(\lambda_{j}(T-\gamma)-2 \theta_{j}\right)=\operatorname{sgn}\left(\sin \left(\lambda_{j} \gamma\right)\right)$ for all $j \in \mathbb{N}^{*}$ and $\left(\rho_{j}\right)_{j \in \mathbb{N}^{*}}$ as a Kronecker delta, and we thus get (24).

In the following lemma, pointed out as a main ingredient of the proof, we establish an energy estimate on $(\eta, T-\eta) \times(\alpha+\eta, \beta-\eta)$ for $\eta \in(0, \beta-\alpha)$.

Lemma 6. Let $\eta \in(0, \beta-\alpha)$ and $T \geqslant 2 \eta$. The energy estimate

$$
\int_{\eta}^{T-\eta} \int_{\alpha+\eta}^{\beta-\eta} E_{k^{2}}(t, x) d x d t \leqslant C(\eta) \int_{0}^{T} \int_{\alpha}^{\beta}\left(\varphi_{t}(t, x)^{2}+\varphi(t, x)^{2}\right) d x d t .
$$

holds with $C(\eta)=\frac{1}{2 \eta}+\max \left\{1, \frac{1}{\eta^{2}}\right\}+\max \left\{1, k^{2}\right\}$.

Proof. Multiplying the identity

$$
\left(\varphi \varphi_{t}\right)_{t}(t, x)-\left(\varphi \varphi_{x}\right)_{x}(t, x)-\varphi_{t}(t, x)^{2}+\varphi_{x}(t, x)^{2}+a(x) \varphi(t, x)^{2}=0
$$

valid for almost every $(t, x) \in(0, T) \times(0, \pi)$, by any smooth function $\zeta \in C_{c}^{\infty}((0, T) \times(\alpha, \beta))$ such that $\zeta \geqslant 0$ on $(0, T) \times(\alpha, \beta)$ and $\zeta=1$ in $[\eta, T-\eta] \times[\alpha+\eta, \beta-\eta]$, we get after integration on $(0, T) \times(0, \pi)$,

$$
\begin{aligned}
\int_{\alpha+\eta}^{\beta-\eta} \int_{\eta}^{T-\eta} \varphi_{x}(t, x)^{2} \zeta(t, x) d t d x \leqslant & \int_{\alpha}^{\beta} \int_{0}^{T} \varphi_{x}(t, x)^{2} \zeta(t, x) d t d x \\
= & \int_{\alpha}^{\beta} \int_{0}^{T}\left(\varphi_{t}(t, x)^{2}-a(x) \varphi(t, x)^{2}\right) \zeta(t, x) d t d x \\
& +\int_{\alpha}^{\beta} \int_{0}^{T}\left(\left(\varphi \varphi_{x}\right)_{x}(t, x)-\left(\varphi \varphi_{t}\right)_{t}(t, x)\right) \zeta(t, x) d t d x .
\end{aligned}
$$

We now estimate the right hand side in this inequality. Integrating by parts, one gets

$$
-\int_{0}^{T} \int_{\alpha}^{\beta}\left(\varphi \varphi_{t}\right)_{t}(t, x) \zeta(t, x) d x d t \leqslant \frac{\left\|\zeta_{t}\right\|_{\infty}}{2} \int_{0}^{T} \int_{\alpha}^{\beta}\left(\varphi_{t}^{2}(t, x)+\varphi^{2}(t, x)\right) d x d t
$$

and

$$
\int_{0}^{T} \int_{\alpha}^{\beta}\left(\varphi \varphi_{x}\right)_{x}(t, x) \zeta(t, x) d x d t \leqslant \frac{\left\|\zeta_{x x}\right\|_{\infty}}{2} \int_{0}^{T} \int_{\alpha}^{\beta} \varphi^{2}(t, x) d x d t .
$$


We then infer

$$
\int_{\alpha+\eta}^{\beta-\eta} \int_{\eta}^{T-\eta} \varphi_{x}(t, x)^{2} d t d x \leqslant c(\eta) \int_{0}^{T} \int_{\alpha}^{\beta}\left(\varphi_{t}(t, x)^{2}+\varphi(t, x)^{2}\right) d x d t
$$

where $c(\eta)=\frac{\left\|\zeta_{t}\right\|_{\infty}}{2}+\max \left\{\|\zeta\|_{\infty}, \frac{\left\|\zeta_{x x}\right\|_{\infty}}{2}\right\}$, for every $\eta \in(0, \beta-\alpha)$. The energy estimate

$$
\int_{\eta}^{T-\eta} \int_{\alpha+\eta}^{\beta-\eta} E_{k^{2}}(t, x) d x d t \leqslant\left(c(\eta)+\max \left\{1,\|a\|_{\infty}\right\}\right) \int_{0}^{T} \int_{\alpha}^{\beta}\left(\varphi_{t}(t, x)^{2}+\varphi(t, x)^{2}\right) d x d t
$$

is thus a consequence of the previous inequalities. Finally, choosing a particular function $\zeta$ enjoying the symmetry properties

$$
\zeta(T-t, x)=\zeta(t, x), \quad \zeta(t, \alpha+y)=\zeta(t, \beta-y)
$$

for every $t \in[0, T]$ and $y \in[0, \beta-\alpha]$, and defined by $\zeta(t, x)=\frac{t}{\eta} \frac{(x-\alpha)^{2}}{\eta^{2}}$ on $[0, \eta] \times[\alpha, \alpha+\eta]$ leads to the expected result.

Remark 11. We stress that the particular choice of function $\zeta$ used above is motivated by the facts that the function $f: t \mapsto t / \eta$ solves the problem

$$
\inf \left\{\left\|f^{\prime}\right\|_{\infty} \mid f \in W^{1, \infty}(0, \eta), f(0)=0 \text { and } f(\eta)=1\right\}
$$

and the function $g: x \mapsto x^{2} / \eta^{2}$ solves the problem

$$
\inf \left\{\left\|g^{\prime \prime}\right\|_{\infty} \mid g \in W^{2, \infty}(0, \eta), g(0)=0 \text { and } g(\eta)=1\right\} .
$$

With the notations of Figure 2, propagations properties of the wave equation are used in the following lemma to derive an energy estimate from $Q_{1}$ to $P_{1}$ and from $Q_{2}$ to $P_{2}$.

Lemma 7. Let $T>T_{0}$ with $T_{0}=2 \max \{\alpha, \pi-\beta\}$ and $\eta \in\left(0, \frac{\beta-\alpha}{4}\right)$. Recall that $C(\eta)$ is the constant defined in the statement of Lemma 6.

i) Introduce the positive number $\delta$ such that $\pi-\beta=\frac{T}{2}-2 \delta$. Then, one has

$$
\int_{\frac{T}{2}-\delta}^{\frac{T}{2}+\delta} \int_{\beta-\eta}^{\pi} E_{k^{2}}(t, x) d x d t \leqslant \frac{C(\eta)}{2 k \eta}\left(e^{2 k(\pi-\beta+3 \eta)}-e^{4 k \eta}\right) \int_{0}^{T} \int_{\alpha}^{\beta}\left(\varphi_{t}^{2}(t, \sigma)+\varphi^{2}(t, \sigma)\right) d \sigma d t,
$$

for every $\eta \in\left(0, \min \left\{\frac{\beta-\alpha}{4}, \frac{\delta}{4}\right\}\right)$.

ii) Introduce the positive number $\delta^{\prime}$ such that $\alpha=\frac{T}{2}-2 \delta^{\prime}$. Then, one has

$$
\int_{\frac{T}{2}-\delta^{\prime}}^{\frac{T}{2}+\delta^{\prime}} \int_{0}^{\alpha+\eta} E_{k^{2}}(t, x) d x d t \leqslant \frac{C(\eta)}{2 k \eta}\left(e^{-2 k \eta}-e^{-2 k(\alpha+2 \eta)}\right) \int_{0}^{T} \int_{\alpha}^{\beta}\left(\varphi_{t}^{2}(t, \sigma)+\varphi^{2}(t, \sigma)\right) d \sigma d t,
$$

for every $\eta \in\left(0, \min \left\{\frac{\beta-\alpha}{4}, \frac{\delta^{\prime}}{4}\right\}\right)$. 
Proof. Let $\xi \in(\alpha, \beta-\eta)$ and $x \in(\xi, \min \{T+\xi, \pi\})$. Using an integration by $\operatorname{parts}^{4}$, one gets

$$
\begin{aligned}
\frac{d}{d x} \int_{x-\xi}^{T-x+\xi} E_{k^{2}}(t, x) d t= & -\left(\varphi_{t}-\varphi_{x}\right)^{2}(T+\xi-x, x)-\left(\varphi_{t}+\varphi_{x}\right)^{2}(x-\xi, x) \\
& -k^{2}\left(\varphi^{2}(T+\xi-x, x)+\varphi^{2}(x-\xi, x)\right)+4 \int_{x-\xi}^{T-(x-\xi)} k^{2} \varphi_{x} \varphi d t, \\
\leqslant & 4 \int_{x-\xi}^{T-(x-\xi)} k^{2}\left|\varphi_{x} \varphi\right| d t .
\end{aligned}
$$

We thus infer that

$$
\frac{d}{d x} \int_{x-\xi}^{T-x+\xi} E_{k^{2}}(t, x) d t \leqslant 2 k \int_{x-\xi}^{T-x+\xi} E_{k^{2}}(t, x) d t .
$$

By noting that the function $x \in(\xi, \pi) \mapsto e^{-2 k(x-\xi-\eta)} \int_{x-\xi}^{T-x+\xi} E_{k^{2}}(t, x) d t$ is non increasing, it follows that

$$
\int_{\frac{T}{2}-\delta}^{\frac{T}{2}+\delta} E_{k^{2}}(t, x) d t \leqslant \int_{x-\xi}^{T-x+\xi} E_{k^{2}}(t, x) d t \leqslant e^{2 k(x-\xi-\eta)} \int_{\eta}^{T-\eta} E_{k^{2}}(t, \xi+\eta) d t,
$$

for every $\eta \in\left(0, \frac{\delta}{4}\right), \xi \in[\beta-4 \eta, \beta-2 \eta]$ and $x \in[\beta-\eta, \pi]$. Integrating this inequality with respect to $\xi$ on $[\beta-4 \eta, \beta-3 \eta]$ yields

$$
\int_{\frac{T}{2}-\delta}^{\frac{T}{2}+\delta} E_{k^{2}}(t, x) d t \leqslant \frac{e^{2 k(x-\beta+3 \eta)}}{\eta} \int_{\eta}^{T-\eta} \int_{\beta-3 \eta}^{\beta-2 \eta} E_{k^{2}}(t, \sigma) d t d \sigma
$$

for every $x \in[\beta-\eta, \pi]$. Notice that $(\beta-3 \eta, \beta-2 \eta) \subset(\alpha+\eta, \beta-\eta)$ since $\eta \leqslant \frac{\beta-\alpha}{4}$. Hence, one deduces the expected result by combining the last inequality with the conclusion of Lemma 6 .

It remains now to prove the second energy estimate. It suffices to mimic the first part of the proof, noting first that

$$
\frac{d}{d x} \int_{\xi-x}^{T-\xi+x} E_{k^{2}}(t, x) d t \geqslant 2 k \int_{\xi-x}^{T-\xi+x} E_{k^{2}}(t, x) d t
$$

for all $\xi \in(\alpha+\eta, \beta)$ and $x \in[0, \xi]$, and second that the function $x \in(0, \xi) \mapsto e^{-2 k(x-\xi+\eta)} \int_{\xi-x}^{T-\xi+x} E_{k^{2}}(t, x) d t$ is non decreasing.

We now prove Theorem 2. With the notations of Lemma 7, introduce $\delta^{-}=\min \left\{\delta, \delta^{\prime}\right\}=\frac{T-T_{0}}{4}$. Combining (25), (26) et (27) yields

$$
\int_{\frac{T}{2}-\delta^{-}}^{\frac{T}{2}+\delta^{-}} \int_{0}^{\pi} E_{k^{2}}(t, x) d x d t \leqslant K(\eta, \alpha, \beta, k) \int_{0}^{T} \int_{\alpha}^{\beta}\left(\varphi_{t}^{2}+\varphi^{2}\right)(t, x) d x d t,
$$

where $K(\eta, \alpha, \beta, k)$ is defined by (11). According to (2), we infer

$$
2 \delta^{-}\left\|\varphi_{t}\left(\frac{T}{2}, \cdot\right)\right\|_{L^{2}(0, \pi)}^{2} \leqslant 2 \delta^{-} \int_{0}^{\pi} E_{a}\left(\frac{T}{2}, x\right) d x \leqslant K(\eta, \alpha, \beta, k) \int_{0}^{T} \int_{\alpha}^{\beta}\left(\varphi_{t}^{2}(t, x)+\varphi^{2}(t, x)\right) d x d t,
$$

\footnotetext{
${ }^{4}$ We also use that, for any function $f$ regular enough, the following identity

$$
\frac{d}{d x} \int_{x-\xi}^{T-x+\xi} f(t, x) d t=-f(T+\xi-x, x)-f(x-\xi, x)+\int_{x-\xi}^{T-x+\xi} \frac{\partial f}{\partial x}(t, x) d t
$$
}

holds for every $x \in(\xi, \min \{T+\xi, \pi\})$. 
for every $\eta \in\left(0, \min \left\{\frac{\delta^{-}}{4}, \frac{\beta-\alpha}{4}\right\}\right)$ and $T>T_{0}$.

It remains now to compare the right hand in (32) with the observation term. Let $\gamma>0$ such that $\gamma \leqslant \delta^{-}$and let $\tau \in\left(\frac{T}{2}-\gamma, \frac{T}{2}\right)$. Introduce the function $\psi$ defined by

$$
\begin{aligned}
\psi:(0,2 \tau) \times(0, \pi) & \rightarrow \mathbb{R} \\
(t, x) & \mapsto \int_{\tau}^{t}\left(\varphi_{t}(s, x)+\varphi_{t}(2 \tau-s, x)\right) d s .
\end{aligned}
$$

Notice that $\psi$ satisfies the main equation of (1) on $(0,2 \tau) \times \Omega$ and that $\psi_{t}(\tau, \cdot)=2 \varphi_{t}(\tau, \cdot)$. Furthermore, the function $\psi$ clearly satisfies the inequality (32). Since $\tau>T_{0} / 2$, we claim

$$
\left\|\psi_{t}(\tau, \cdot)\right\|_{L^{2}(0, \pi)}^{2} \leqslant \frac{K(\eta, \alpha, \beta, k)}{2 \mu^{-}} \int_{0}^{2 \tau} \int_{\alpha}^{\beta}\left(\psi_{t}^{2}+\psi^{2}\right)(t, x) d x d t
$$

for every $\eta \in\left(0, \min \left\{\frac{\mu^{-}}{4}, \frac{\beta-\alpha}{4}\right\}\right)$, where $\mu^{-}=\min \left\{\mu, \mu^{\prime}\right\}$ with $\mu=\frac{\tau-\pi+\beta}{2}$, and $\mu^{\prime}=\frac{\tau-\alpha}{2}$. We thus infer that

$$
\begin{aligned}
\frac{8 \mu^{-}}{K(\eta, \alpha, \beta, k)}\left\|\varphi_{t}(\tau, \cdot)\right\|_{L^{2}(0, \pi)}^{2} \leqslant & \int_{0}^{2 \tau} \int_{\alpha}^{\beta}\left(\varphi_{t}(t, x)+\varphi_{t}(2 \tau-t, x)\right)^{2} d x d t \\
& +\int_{0}^{2 \tau} \int_{\alpha}^{\beta}\left(\int_{\tau}^{t} \varphi_{t}(s, x)+\varphi_{t}(2 \tau-s, x) d s\right)^{2} d x d t .
\end{aligned}
$$

Introducing

$$
R_{\tau}=\int_{0}^{2 \tau}\left(\int_{\tau}^{t}\left(\varphi_{t}(s, x)+\varphi_{t}(2 \tau-s, x)\right) d s\right)^{2} d t
$$

one gets using the Cauchy-Schwarz inequality

$$
\begin{aligned}
\frac{R_{\tau}}{2} \leqslant & \int_{0}^{\tau}(\tau-t) \int_{t}^{\tau} \varphi_{t}^{2}(s, x) d s d t+\int_{\tau}^{2 \tau}(t-\tau) \int_{\tau}^{t} \varphi_{t}^{2}(s, x) d s d t \\
& +\int_{0}^{\tau}(\tau-t) \int_{t}^{\tau} \varphi_{t}^{2}(2 \tau-s, x) d s d t+\int_{\tau}^{2 \tau}(t-\tau) \int_{\tau}^{t} \varphi_{t}^{2}(2 \tau-s, x) d s d t, \\
\leqslant & \tau^{2} \int_{0}^{2 \tau} \varphi_{t}^{2}(s, x) d s,
\end{aligned}
$$

and eventually

$$
\left\|\varphi_{t}(\tau, \cdot)\right\|_{L^{2}(0, \pi)}^{2} \leqslant \frac{K(\eta, \alpha, \beta, k)}{2 \mu^{-}}\left(1+\frac{\tau^{2}}{2}\right) \int_{0}^{2 \tau} \int_{\alpha}^{\beta} \varphi_{t}^{2}(t, x) d x d t .
$$

Let us now provide an estimate of the right-hand side in the last inequality that is uniform with respect to $\tau$. Using that $\tau \in\left(\frac{T}{2}-\gamma, \frac{T}{2}\right)$,

$$
\mu \geqslant \frac{\frac{T}{2}-\gamma-c+\beta}{2}, \quad \mu^{\prime} \geqslant \frac{\frac{T}{2}-\gamma-\alpha}{2} \text { and } \mu^{-} \geqslant \frac{T-T_{0}}{4}-\frac{\gamma}{2}
$$

and that the expression of $K(\eta, \alpha, \beta, k)$ above makes sense for $\eta \in\left(0, \min \left\{\frac{T-T_{0}}{16}-\frac{\gamma}{8}, \frac{\beta-\alpha}{4}\right\}\right)$, we get

$$
\int_{\frac{T}{2}-\gamma}^{\frac{T}{2}}\left\|\varphi_{t}(\tau, \cdot)\right\|_{L^{2}(0, \pi)}^{2} d \tau \leqslant \frac{2 \gamma K(\eta, \alpha, \beta, k)}{T-T_{0}-2 \gamma}\left(1+\frac{T^{2}}{8}\right) \int_{0}^{T} \int_{\alpha}^{\beta} \varphi_{t}^{2}(t, x) d x d t .
$$


Applying Lemma 5 leads to

$$
\left(\frac{\gamma}{2}-\sup _{j \in \mathbb{N}^{*}} \frac{\left|\sin \left(\lambda_{j} \gamma\right)\right|}{2 \lambda_{j}}\right) \frac{4\left(T-T_{0}-2 \gamma\right)}{\left(8+T^{2}\right) \gamma K(\eta, \alpha, \beta, k)} \leqslant \frac{\int_{0}^{T} \int_{\alpha}^{\beta} \varphi_{t}^{2}(t, x) d x d t}{\int_{0}^{\pi} E_{a}(0, x) d x},
$$

providing hence a lower estimate of the observability constant.

For large values of $T$, it is yet possible to improve this estimate. Assuming that $T \geqslant 2 T_{0}$, we write

$$
\int_{0}^{T} \int_{\alpha}^{\beta} \varphi_{t}(t, x)^{2} d x d t \geqslant \int_{0}^{2 T_{0}\left[\frac{T}{2 T_{0}}\right]} \int_{\alpha}^{\beta} \varphi_{t}(t, x)^{2} d x d t=\sum_{i=0}^{\left[\frac{T}{2 T_{0}}\right]-1} \int_{2 i T_{0}}^{2(i+1) T_{0}} \int_{\alpha}^{\beta} \varphi_{t}(t, x)^{2} d x d t
$$

and using the time reversibility of the wave equation, the inequality (33) improves into

$$
\left[\frac{T}{2 T_{0}}\right]\left(\frac{\gamma}{2}-\sup _{j \in \mathbb{N}^{*}} \frac{\left|\sin \left(\lambda_{j} \gamma\right)\right|}{2 \lambda_{j}}\right) \frac{\left(T_{0}-2 \gamma\right)}{\left(2+T_{0}^{2}\right) \gamma K(\eta, \alpha, \beta, k)} \leqslant \frac{\int_{0}^{T} \int_{\alpha}^{\beta} \varphi_{t}^{2}(t, x) d x d t}{\int_{0}^{\pi} E_{a}(0, x) d x} .
$$

\subsection{Proof of Corollary 1}

Notice that, in the proof of Theorem 2, we only made local reasonings around the observation open set $\omega$, and we never used the Dirichlet boundary conditions. Considering $\varphi$, the unique solution of the wave equation (1) with initial data $\left(\varphi(0, \cdot), \partial_{t} \varphi(0, \cdot)\right)=\left(\varphi_{0}, \varphi_{1}\right) \in H_{0}^{1}(0, \pi) \times L^{2}(0, \pi)$. Let us apply the estimate of the observability constant proved for one open interval in Section 2.2 on $(0, T) \times\left(\beta_{i-1}, \alpha_{i+1}\right)$, we obtain using the notations of Theorem 2 and replacing (11) by (14)

$$
C_{T, T_{0}, a, \lambda}^{\prime}\left(\alpha_{i}, \beta_{i}\right) \int_{\beta_{i-1}}^{\alpha_{i+1}} E_{a}(0, x) d x \leqslant \int_{0}^{T} \int_{\alpha_{i}}^{\beta_{i}} \varphi_{t}(t, x)^{2} d x d t
$$

for every $i=1, \cdots, n$. Since $\int_{0}^{\pi} E_{a}(0, x) d x \leqslant \sum_{i=1}^{n} \int_{\beta_{i-1}}^{\alpha_{i+1}} E_{a}(0, x) d x$, it follows that

$$
\min _{1 \leqslant i \leqslant n} C_{T, T_{0}, a, \lambda}^{\prime}\left(\alpha_{i}, \beta_{i}\right) \int_{0}^{\pi} E_{a}(0, x) d x \leqslant \int_{0}^{T} \int_{\omega} \varphi_{t}(t, x)^{2} d x d t,
$$

whence the conclusion.

\subsection{Proof of Corollary 2}

Assume in a first time that $\omega=(\alpha, \beta)$. We follow the same lines as in the proof of Theorem 2, and propose a different way to conclude from the inequality (31). For this reason, we only provide here some explanations to adapt the proof of Theorem 2 to this simpler case.

Let us decompose the solution $\varphi$ of (1) in the spectral basis $\left\{e_{j}\right\}_{j \in \mathbb{N}^{*}}$ as in (5)-(6). Since $j_{0}=\left[\frac{\|a\|_{\infty}-1}{2}\right]+1$, one gets by adapting the proof of Lemma 2 ,

$$
\lambda_{j+1}-\lambda_{j} \geqslant \frac{1+2 j_{0}-\|a\|_{\infty}}{j_{0}+1+\sqrt{j_{0}^{2}+\|a\|_{\infty}}}>0, \quad \text { for every } j \geqslant j_{0} .
$$

Thus, $\lambda_{j+1}-\lambda_{j} \geqslant \gamma_{j_{0}}$ for every $j \in \mathbb{N}^{*}$. 
Thus, applying Ingham's inequalities (see [10] and Remark 5) leads to

$$
\begin{aligned}
\int_{0}^{T} \int_{\omega} \varphi(t, x)^{2} d x d t & =\frac{1}{2} \int_{0}^{T} \int_{\omega}\left|\sum_{k \in \mathbb{Z}^{*}} \sqrt{a_{|k|}^{2}+b_{|k|}^{2}} e^{i \operatorname{sgn}(k)\left(\lambda_{|k|} t-\theta_{|k|}\right)} e_{|k|}(x)\right|^{2} d x d t \\
& \leqslant \frac{10 T}{\min \left\{2 \pi, \gamma_{j_{0}} T\right\}} \sum_{j=1}^{+\infty}\left(a_{j}^{2}+b_{j}^{2}\right) \int_{\omega} e_{j}(x)^{2} d x
\end{aligned}
$$

and

$$
\begin{aligned}
\int_{0}^{T} \int_{\omega}\left|\partial_{t} \varphi(t, x)\right|^{2} d x d t & =\frac{1}{2} \int_{0}^{T} \int_{\omega}\left|\sum_{k \in \mathbb{Z}^{*}} i \operatorname{sgn}(k) \lambda_{|k|} \sqrt{a_{|k|}^{2}+b_{|k|}^{2}} e^{i \operatorname{sgn}(k)\left(\lambda_{|k|} t-\theta_{|k|}\right)} e_{|k|}(x)\right|^{2} d x d t \\
& \geqslant \frac{1}{\pi}\left(T-\frac{4 \pi^{2}}{\gamma_{j_{0}}^{2} T}\right) \sum_{j=1}^{+\infty} \lambda_{j}^{2}\left(a_{j}^{2}+b_{j}^{2}\right) \int_{\omega} e_{j}(x)^{2} d x
\end{aligned}
$$

for every $T>T_{0}^{\prime}$. Using (19), one gets

$$
\int_{0}^{T} \int_{\omega} \varphi(t, x)^{2} d x d t \leqslant \frac{10 \pi \gamma_{j_{0}}^{2} T^{2}}{\left(\gamma_{j_{0}}^{2} T^{2}-4 \pi^{2}\right) \min \left\{2 \pi, \gamma_{j_{0}} T\right\}} \int_{0}^{T} \int_{\omega}\left|\partial_{t} \varphi(t, x)\right|^{2} d x d t .
$$

Combining this inequality with (32) yields

$$
\begin{aligned}
\frac{\left(T-T_{0}^{\prime}\right)\left(\gamma_{j_{0}}^{2} T^{2}-4 \pi^{2}\right) \min \left\{2 \pi, \gamma_{j_{0}}, T\right\}}{K\left(\eta, \alpha, \beta,\|a\|_{\infty}^{1 / 2}\right)\left(2\left(\gamma_{j_{0}}^{2} T^{2}-4 \pi^{2}\right) \min \left\{2 \pi, \gamma_{j_{0}}, T\right\}+20 \pi \gamma_{j_{0}}^{2} T^{2}\right)} \int_{0}^{\pi} E_{a}(0, x) d x \\
\leqslant \int_{0}^{T} \int_{\alpha}^{\beta} \varphi_{t}(t, x)^{2} d x d t,
\end{aligned}
$$

for every $\eta \in\left(0, \min \left\{\frac{T-T_{0}}{16}, \frac{\beta-\alpha}{4}\right\}\right)$, providing an estimate similar to (33) in this case. The conclusion follows, adapting the proof of Corollary 1 to extend the results to observation domains $\omega$ that are the finite union of open intervals.

\section{Applications}

\subsection{Extension of the previous results to general wave equations}

Let $\ell$ and $T$ denote two positive constants. We consider the general one dimensional wave equation with Dirichlet boundary conditions

$$
\begin{array}{ll}
\partial_{t t} \phi(t, z)-\partial_{z}\left(b(z) \partial_{z} \phi\right)(t, z)+\bar{a}(z) \phi(t, z)=0 & (t, z) \in(0, T) \times(0, \ell), \\
\phi(t, 0)=\phi(t, \ell)=0 & t \in[0, T], \\
\phi(0, z)=\phi_{0}(z), \partial_{t} \phi(0, z)=\phi_{1}(z) & z \in[0, \ell],
\end{array}
$$

where it is assumed that $b$ and $\bar{a}$ denote nonnegative functions in $L^{\infty}(0, \ell)$ and that there exist a constant $b_{0}>0$ such that $b \geqslant b_{0}$ a.e. in $(0, \ell)$.

Recall that for every initial data $\left(\phi_{0}, \phi_{1}\right) \in H_{0}^{1}(0, \ell) \times L^{2}(0, \ell)$, Equation (35) has a unique solution $\phi \in C^{0}\left(0, T ; H_{0}^{1}(0, \ell)\right) \cap C^{1}\left(0, T ; L^{2}(0, \ell)\right)$. Moreover, the equation (35) is said to be observable on a measurable subset $\omega \subset(0, \ell)$ in time $T$ if there exists a positive constant $c$ such that

$$
\int_{0}^{T} \int_{\omega} \partial_{t} \phi(t, z)^{2} d z d t \geqslant c \int_{0}^{\ell}\left(\phi_{1}(z)^{2}+b(z) \phi_{0}^{\prime}(z)^{2}+\bar{a}(z) \phi_{0}(x)^{2}\right) d z .
$$


Let us denote by $\bar{c}(T, \ell, b, \bar{a}, \omega)$ the best constant in this inequality, that is

$$
\bar{c}(T, \ell, b, \bar{a}, \omega)=\inf _{\substack{\left(\phi_{0}, \phi_{1}\right) \in H_{0}^{1}(0, \ell) \times L^{2}(0, \ell) \\\left(\phi_{0}, \phi_{1}\right) \neq(0,0)}} \frac{\int_{0}^{T} \int_{\omega} \partial_{t} \phi(t, z)^{2} d z d t}{\int_{0}^{\ell}\left(\phi_{1}(z)^{2}+b(z) \varphi_{0}^{\prime}(z)^{2}+\bar{a}(z) \varphi_{0}(z)^{2}\right) d z} .
$$

Notice in particular that, with the notations of Section 1, one has $c(T, a, \omega)=\bar{c}(T, \pi, \operatorname{Id}, a, \omega)$.

The following result highlights the link between the observability of (35) and the observability of (1). For that purpose, let us introduce the standard change of variable for Sturm-Liouville equations

$$
X: z \longmapsto \frac{\pi}{\ell^{\prime}} \int_{0}^{z} \frac{d s}{\sqrt{b(s)}}
$$

with $\ell^{\prime}=\int_{0}^{\ell} \frac{d s}{\sqrt{b(s)}}$. Since $b \geqslant b_{0}$ a.e in $(0, \ell)$, the function $X$ is continuous nondecreasing and defines thus a change of variable.

Proposition 1. Let us assume that the function $b$ belongs to $b \in W^{2, \infty}(0, \ell)$, let $\ell^{\prime}=\int_{0}^{\ell} \frac{d s}{\sqrt{b(s)}}$ and $T^{\prime}=\frac{\pi}{\ell^{\prime}} T$. We define the function $a(\cdot)$ by

$$
a(x)=\frac{\ell^{\prime 2}}{\pi^{2}}\left(\bar{a}(z)-\frac{1}{16} \frac{b^{\prime}(z)^{2}}{b(z)}+\frac{1}{4} b^{\prime \prime}(z)\right),
$$

for almost every $z \in(0, \ell)$ is nonnegative. Thus,

$$
\bar{c}(T, \ell, b, \bar{a}, \omega)=\frac{\ell^{\prime}}{\pi} c\left(T^{\prime}, a, X(\omega)\right)
$$

Proof. Using the change of variable $t^{\prime}=\frac{\pi}{\ell^{\prime}} t$ and introducing $\tilde{\phi}\left(t^{\prime}, \cdot\right)=\phi(t, \cdot)$, the main equation of (35) rewrites

$$
\partial_{t^{\prime} t^{\prime}} \tilde{\phi}\left(t^{\prime}, z\right)-\partial_{z}\left(\tilde{b}(z) \partial_{z} \tilde{\phi}\right)(t, z)+\tilde{a}(z) \tilde{\phi}(t, z)=0, \quad\left(t^{\prime}, z\right) \in\left(0, T^{\prime}\right) \times(0, \ell),
$$

where $\tilde{b}(z)=\frac{\ell^{\prime 2}}{\pi^{2}} b(z)$ and $\tilde{a}(z)=\frac{\ell^{\prime 2}}{\pi^{2}} \bar{a}(z)$. Provided that $b \geqslant b_{0}$ and (37) are verified, $\phi$ is solution of (35) if and only if the function $\varphi:\left(t^{\prime}, x\right) \mapsto \tilde{b}(z)^{1 / 4} \tilde{\phi}\left(t^{\prime}, z\right)$ is solution of (1). The result follows, by writing that

$$
\begin{aligned}
\frac{\pi}{\ell^{\prime}} \frac{\int_{0}^{T} \int_{\omega} \partial_{t} \phi(t, z)^{2} d z d t}{\int_{0}^{\ell}\left(\phi_{1}(z)^{2}+b(z) \phi_{0}^{\prime}(z)^{2}+\bar{a}(z) \phi_{0}(z)^{2}\right) d z} & =\frac{\int_{0}^{T^{\prime}} \int_{\omega} \partial_{t^{\prime}} \tilde{\phi}\left(t^{\prime}, z\right)^{2} d z d t}{\int_{0}^{\ell}\left(\tilde{\phi}_{t^{\prime}}(0, z)^{2}+\tilde{b}(z) \tilde{\phi}_{z}(0, z)^{2}+\tilde{a}(z) \tilde{\phi}(0, z)^{2}\right) d z} \\
& =\frac{\int_{0}^{T^{\prime}} \int_{X(\omega)} \partial_{t^{\prime}} \varphi\left(t^{\prime}, x\right)^{2} d x d t}{\int_{0}^{\pi}\left(\varphi_{t^{\prime}}(0, x)^{2}+\varphi_{x}(0, x)^{2}+a(x) \varphi(0, x)^{2}\right) d x}
\end{aligned}
$$

Using the correspondence between the observability of Equations (35) and (1), it is easy to deduce estimates of $\bar{c}(T, \ell, b, \bar{a}, \omega)$ from the observability constants estimates in Theorems 1,2 , Corollaries 1 and 2, provided that the assumptions above be verified. 


\subsection{Evaluating the cost of control for the Hilbert Uniqueness Method.}

Consider the internally controlled wave equation on $(0, \pi)$ with Dirichlet boundary conditions

$$
\begin{array}{ll}
\partial_{t t} y(t, x)-\partial_{x x} y(t, x)+a(x) y(t, x)=h_{\omega}(t, x) & (t, x) \in(0, T) \times(0, \pi), \\
y(t, 0)=y(t, \pi)=0 & t \in[0, T], \\
y(0, x)=y^{0}(x), \partial_{t} y(0, x)=y^{1}(x) & x \in(0, \pi),
\end{array}
$$

where $h_{\omega}$ is a control supported in $[0, T] \times \omega$ where $\omega \subset(0, \pi)$ is Lebesgue measurable. Recall that for all initial data $\left(y^{0}, y^{1}\right) \in H_{0}^{1}(0, \pi) \times L^{2}(0, \pi)$ and every $h_{\omega} \in L^{2}((0, T) \times(0, \pi))$, the problem (38) is well posed and its solution $y$ belongs to $C^{0}\left(0, T ; H_{0}^{1}(0, \pi)\right) \cap C^{1}\left(0, T ; L^{2}(0, \pi)\right) \cap$ $C^{2}\left(0, T ; H^{-1}(0, \pi)\right)$.

In what follows, we will endow the space $H_{0}^{1}(0, \pi)$ with the inner product

$$
\left(H_{0}^{1}(0, \pi)\right)^{2} \ni(\varphi, \psi) \longmapsto \int_{0}^{\pi} \varphi^{\prime}(x) \psi^{\prime}(x) d x+\int_{0}^{\pi} a(x) \varphi(x) \psi(x) d x,
$$

instead of the standard inner-product of $H^{1}(0, \pi)$, according to the choice of norm in the energy space made to introduce the inequality (3). Therefore, the space $H^{-1}(0, \pi)$ will be endowed with the dual norm.

The exact null controllability problem settled in these spaces consists of finding a control $h_{\omega}$ steering the control system $(38)$ to $y(T, \cdot)=\partial_{t} y(T, \cdot)=0$. It is well known that the Hilbert Uniqueness Method (HUM, see $[16,17])$ provides a way to design the unique control solving the above exact null controllability problem and having moreover a minimal $L^{2}((0, T) \times(0, \pi))$ norm.

Using the observability inequality

$$
\int_{0}^{T} \int_{\omega} \varphi(t, x)^{2} d x d t \geqslant c\left\|\left(\varphi^{0}, \varphi^{1}\right)\right\|_{L^{2}(0, \pi) \times H^{-1}(0, \pi)}^{2},
$$

where $c$ is a positive constant (only depending on $T$ and $\omega$ ), valuable for every solution $\varphi$ of the adjoint system

$$
\begin{array}{ll}
\partial_{t t} \varphi(t, x)-\partial_{x x} \varphi(t, x)+a(x) \varphi(t, x)=0, & (t, x) \in(0, T) \times(0, \pi), \\
\varphi(t, 0)=\varphi(t, \pi)=0, & t \in[0, T], \\
\varphi(0, x)=\varphi^{0}(x), \partial_{t} \varphi(0, x)=\varphi^{1}(x), & x \in[0, \pi],
\end{array}
$$

and every $T \geqslant 2 \pi$, the functional

$$
J_{\omega}\left(\varphi^{0}, \varphi^{1}\right)=\frac{1}{2} \int_{0}^{T} \int_{\omega} \varphi(t, x)^{2} d x d t-\left\langle\varphi^{1}, y^{0}\right\rangle_{H^{-1}, H_{0}^{1}}+\left\langle\varphi^{0}, y^{1}\right\rangle_{L^{2}},
$$

has a unique minimizer (still denoted $\left.\left(\varphi^{0}, \varphi^{1}\right)\right)$ in the space $L^{2}(0, \pi) \times H^{-1}(0, \pi)$, for all $\left(y^{0}, y^{1}\right) \in$ $H_{0}^{1}(0, \pi) \times L^{2}(0, \pi)$. In (41) the notation $\langle\cdot, \cdot\rangle_{H^{-1}, H_{0}^{1}}$ stands for the duality bracket between $H^{-1}(0, \pi)$ and $H_{0}^{1}(0, \pi)$, and the notation $\langle\cdot, \cdot\rangle_{L^{2}}$ stands for the usual scalar product of $L^{2}(0, \pi)$. The HUM control $h_{\omega}$ steering $\left(y^{0}, y^{1}\right)$ to $(0,0)$ in time $T$ is then given by

$$
h_{\omega}(t, x)=\chi_{\omega}(x) \varphi(t, x),
$$

for almost all $(t, x) \in(0, T) \times(0, \pi)$, where $\chi_{\omega}$ denotes the characteristic function of the measurable set $\omega$ and $\varphi$ is the solution of (40) with initial data $\left(\varphi^{0}, \varphi^{1}\right)$ minimizing $J_{\omega}$.

The HUM operator $\Gamma_{\omega}$ is then defined by

$$
\begin{aligned}
\Gamma_{\omega}: H_{0}^{1}(0, \pi) \times L^{2}(0, \pi) & \longrightarrow L^{2}((0, T) \times(0, \pi)) \\
\left(y^{0}, y^{1}\right) & \longmapsto h_{\omega}
\end{aligned}
$$


and the norm of the operator $\Gamma_{\omega}$ is given by

$$
\left\|\Gamma_{\omega}\right\|=\sup \left\{\frac{\left\|h_{\omega}\right\|_{L^{2}((0, T) \times(0, \pi)}}{\left\|\left(y^{0}, y^{1}\right)\right\|_{H_{0}^{1}(0, \pi) \times L^{2}(0, \pi)}} \mid\left(y^{0}, y^{1}\right) \in H_{0}^{1}(0, \pi) \times L^{2}(0, \pi) \backslash\{(0,0)\}\right\} .
$$

Recall that, by duality, the best constant in (39) coincides with the constant $c(T, a, \omega)$ defined by (4), that is the optimal constant in the observability inequality (3).

Proposition 2. Let $T>0$ and let $\omega$ be measurable subset of $(0, \pi)$. If $c(T, a, \omega)>0$ then

$$
\left\|\Gamma_{\omega}\right\|=\frac{1}{c(T, a, \omega)}
$$

and if $c(T, a, \omega)=0$, then $\left\|\Gamma_{\omega}\right\|=+\infty$.

For a proof of this result, we refer for instance to [5, 22]. As a consequence, the results in Theorems 1, 2, Corollaries 1 and 2 permit to provide an estimate of the cost of control given by the Hilbert Uniqueness Method.

\subsection{Estimating the stabilization rate of the damped wave equation.}

Consider the damped wave equation on $(0, \pi)$ with Dirichlet boundary conditions

$$
\begin{array}{ll}
\partial_{t t} y(t, x)-\partial_{x x} y(t, x)+2 k_{\omega}(x) \partial_{t} y(t, x)=0 & (t, x) \in(0, T) \times(0, \pi), \\
y(t, 0)=y(t, \pi)=0 & t \in[0, T], \\
y(0, x)=y_{0}(x), \partial_{t} y(0, x)=y_{1}(x) & x \in(0, \pi),
\end{array}
$$

with $k>0$. Recall that for all initial data $\left(y_{0}, y_{1}\right) \in H_{0}^{1}(0, \pi) \times L^{2}(0, \pi)$, the problem (43) is well posed and its solution $y$ belongs to $C^{0}\left(0, T ; H_{0}^{1}(0, \pi)\right) \cap C^{1}\left(0, T ; L^{2}(0, \pi)\right)$.

The energy associated to System (43) is defined by

$$
E(t)=\int_{0}^{\pi}\left(\partial_{t} y(t, x)^{2}+\partial_{x} y(t, x)^{2}\right) d x
$$

According to [3, 4], if $\omega$ has positive measure, this system is exponentially stable, i.e. its energy is known to obey

$$
E(t) \leqslant C E(0) e^{-\delta t}
$$

for $t>0$, where $C$ and $\delta$ denote positive constant that do not depend on the initial data. We define the decay rate $\delta(k, \omega)$ as the largest such $\delta$, in other words

$$
\begin{gathered}
\delta(k, \omega)=\sup \left\{\delta \mid \exists C>0 \text { such that } E(t) \leqslant C E(0) e^{-\delta t}, \text { for every } t>0\right. \\
\text { and for every solution of }(43)\} .
\end{gathered}
$$

According to the following proposition, one can provide an estimate of the constants $C$ and $\delta$ (or $\delta(k, \omega))$ from the estimates proved in Theorems 1, 2, Corollaries 1 and 2.

Proposition 3. Let $\omega$ be a measurable subset of $(0, \pi)$ and $T_{0}$ denotes the minimal observability time $e^{5}$. Thus, for every $\left(y_{0}, y_{1}\right) \in H_{0}^{1}(0, \pi) \times L^{2}(0, \pi)$ and $t \geqslant 2 T_{0}$,

$$
E(t) \leqslant E(0) e^{-\delta t}
$$

with

$$
\delta=\frac{1}{2 T_{0}} \ln \left(\frac{1+\left(1+T_{0}^{2}\right) c\left(T_{0}, a, \omega\right)}{\left(1+T_{0}^{2}\right) c\left(T_{0}, a, \omega\right)}\right)
$$

the constant $c\left(T_{0}, a, \omega\right)$ denoting the observability constant defined by (4).

For a proof of this result, we refer for instance to [8].

\footnotetext{
${ }^{5}$ For instance, if $\omega=(\alpha, \beta)$ one has $T_{0}=2 \max \{\alpha, \pi-\beta\}$
} 


\section{Examples and numerical illustrations}

We provide here some numerical simulations and illustrations of observability constants estimates using both methods (spectral versus propagation).

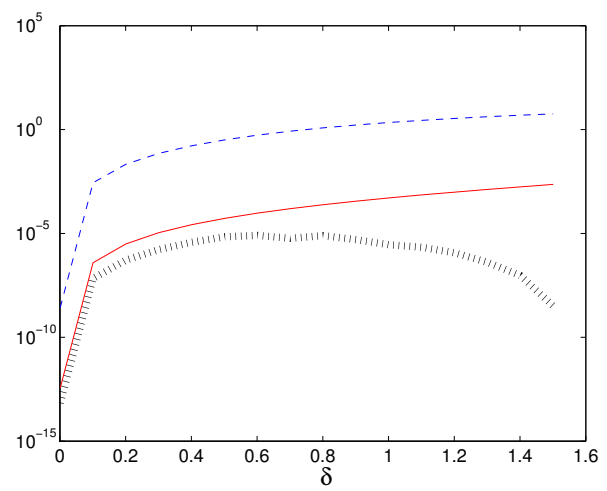

(a) $T=4 \pi$

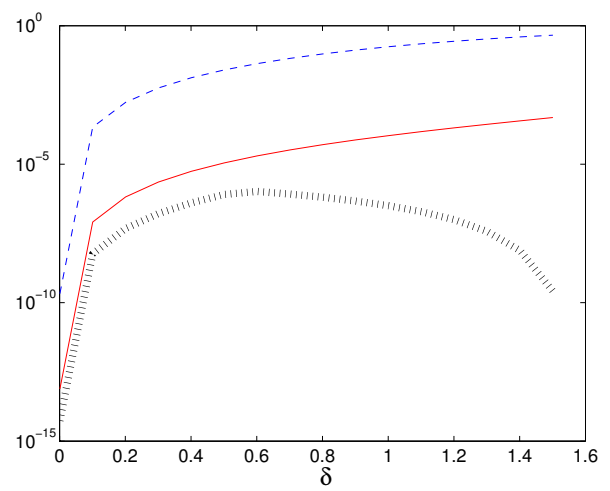

(b) Asymptotic behavior in $T$

Figure 3: $a()=$.0 and $\omega=(0, \delta) \cup(\pi-\delta, \pi)$. Plots of $C_{0}(T,|\omega|)(--), C_{T, T_{0}, 0, \lambda}^{\prime}(/ /)$ and $\widetilde{C}_{T, T_{0}^{\prime}, 0, \lambda}$ $(-)$ with respect to $\delta$

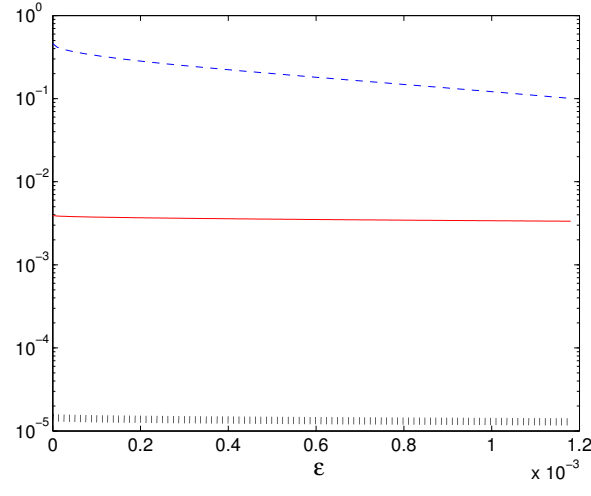

(a) Small values of $\varepsilon$

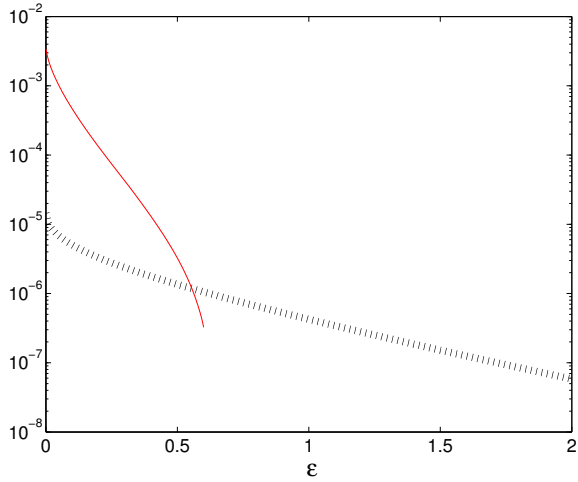

(b) Large values of $\varepsilon$

Figure 4: $a: x \mapsto \varepsilon x, \omega=\left(0, \frac{3}{2}\right)$ and $T=8 \pi$. Plots of $C_{a}(T,|\omega|)(--), C_{T, T_{0}, a, \lambda}^{\prime}(/ /)$ and $\widetilde{C}_{T, T_{0}^{\prime}, a, \lambda}$ $(-)$ with respect to $\varepsilon$

The case $a(\cdot)=0$ is investigated on Figure 3. We chose as observation subset $\omega=(0, \delta) \cup$ 
$(\pi-\delta, \pi)$ with $0 \leqslant \delta \leqslant \pi$. In that case, the observability constant can be explicitly computed ${ }^{6}$ whenever $T$ is a multiple of $2 \pi$, as well as the limit of $c(T, a, \omega) / T$. To compute the estimates (12) and (13), we fully use Remark 9.

More precisely,

- on Figure 3(a), the observation time is $T=4 \pi$. For such a choice of time $T$, the estimate $C_{0}(T,|\omega|)$ obtained by the spectral method coincides with the value of the observability constant. The graphs of the estimates obtained by each method, namely $C_{T, T_{0}, 0, \lambda}^{\prime}$ defined by (13) and $\widetilde{C}_{T, T_{0}^{\prime}, 0, \lambda}$ defined by (15), are represented with respect to the parameter $\delta$ and compared with the observability constant $C_{0}(T,|\omega|)$.

- on Figure 3(b), the graphs of the limit of the estimates as $T$ goes to $+\infty$ obtained by each method, namely $\frac{C_{T, T_{0}, 0, \lambda}^{\prime}}{T}$ and $\frac{\widetilde{C}_{T, T_{0}^{\prime}, 0, \lambda}}{T}$, are represented with respect to the parameter $\delta$ and compared with the limit of the observability constant $\frac{C_{0}(T,|\omega|)}{T}$ as $T$ goes to $+\infty$.

In accordance with Figure 3, one can easily show that the quantity $C_{T, T_{0}, a, \lambda}^{\prime}$ decreases whenever the measure of $\omega$ is large enough, which is a confirmation that this estimate is not sharp.

On Figure 4, we consider the potential $a: x \mapsto \varepsilon x$ with $\varepsilon>0$, the observation set $\omega=\left(0, \frac{3}{2}\right)$ and the observation time $T=8 \pi$. More precisely, the graphs of the estimates obtained by each method, namely $C_{a}(T,|\omega|)$ defined by (8), $C_{T, T_{0}, a, \lambda}^{\prime}$ defined by (13) and $\widetilde{C}_{T, T_{0}^{\prime}, a, \lambda}$ defined by (15), are represented with respect to the parameter $\varepsilon$. On Figure 4(b), only the graphs of $C_{T, T_{0}, a, \lambda}^{\prime}$ (propagation method, Theorem 2) and $\widetilde{C}_{T, T_{0}^{\prime}, a, \lambda}$ (propagation method, Corollary 2) are plotted because of the smallness of the range of $\varepsilon$ for which the spectral method makes sense (due to Condition (7)). Moreover, the range of $\varepsilon$ for which $\widetilde{C}_{T, T_{0}^{\prime}, a, \lambda}$ is defined is restricted, since we use Ingham's inequalities (see the proof of Corollary 2 for more details).

According to Figures 3 and 4(a), the spectral method seems more accurate than propagation methods, but needs the strong condition (7) on the potential function $a(\cdot)$ (see the comments and comparison between both methods at the end of the previous section).

We provide on the figures 5, 6 and 7 several other examples illustrating each method.

${ }^{6}$ Let $\omega=(0, \delta) \cup(\pi-\delta, \pi)$ and $T$ is a multiple of $2 \pi$. The observability constant is exactly

$$
c(T, a, \omega)=C_{0}(T,|\omega|)=\left[\frac{T}{2 \pi}\right](2 \delta-\sin (2 \delta)),
$$

and one has the asymptotic expansion

$$
c(T, a, \omega) \sim \frac{(2 \delta-\sin (2 \delta))}{2 \pi} T .
$$

as $T \rightarrow+\infty$. 


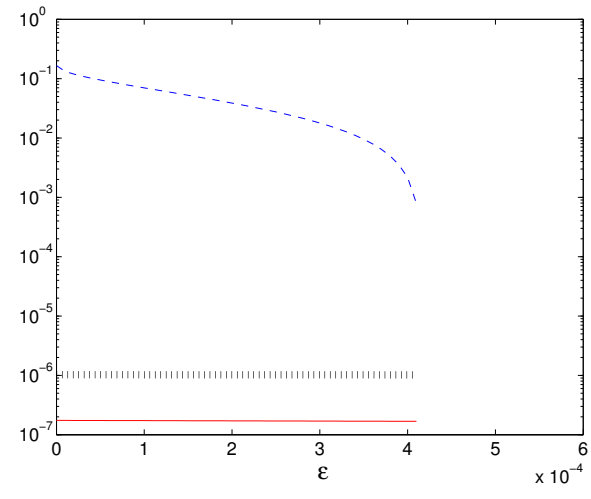

(a) Small positive values of $\varepsilon$

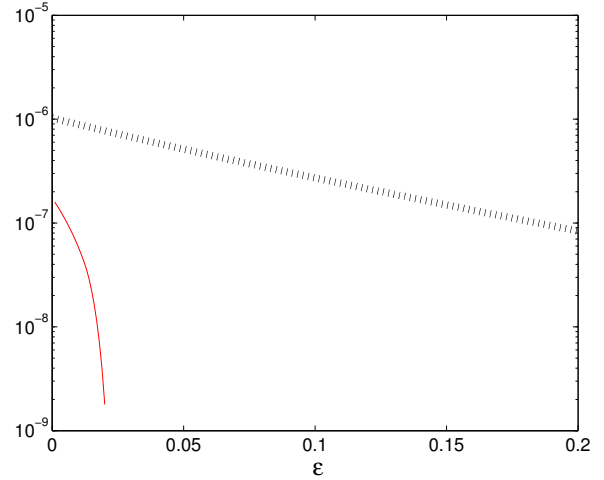

(b) Large positive values of $\varepsilon$

Figure 5: $a: x \mapsto 2+\varepsilon x^{2}, \omega=(0, \pi / 3)$ and $T=10 \pi$. Plots of $C_{0}(T,|\omega|)(--), C_{T, T_{0}, 0, \lambda}^{\prime}(/ /)$ and $\widetilde{C}_{T, T_{0}^{\prime}, 0, \lambda}(-)$ with respect to $\varepsilon$

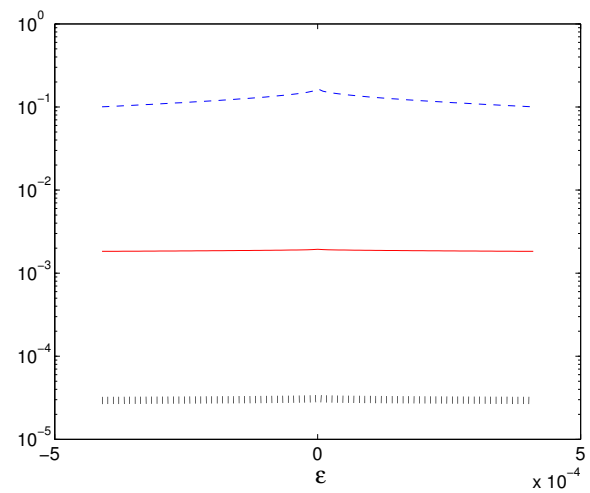

Figure 6: $a: x \mapsto \varepsilon \cos x, \omega=\left(0, \frac{\pi}{3}\right)$ and $T=10 \pi$. Plots of $C_{a}(T,|\omega|)(--), C_{T, T_{0}, a, \lambda}^{\prime}(/ /)$ and $\widetilde{C}_{T, T_{0}^{\prime}, a, \lambda}(-)$ with respect to $\varepsilon$

\section{References}

[1] J. M. Ball, M. Slemrod, Nonharmonic Fourier series and the stabilization of distributed semilinear control systems, Comm. Pure Appl. Math. 32 (1979), no. 4, 555-587.

[2] C. Bardos, G. Lebeau, J. Rauch, Sharp sufficient conditions for the observation, control, and stabilization of waves from the boundary, SIAM J. Control Optim. 30 (1992), no. 05, 10241065 .

[3] G. Chen, S.A. Fulling, F.J. Narcowich and S. Sun, Exponential decay of energy of evolution equations with locally distributed damping, SIAM J. Appl. Math. 51 (1991), no. 1, 266-301. 


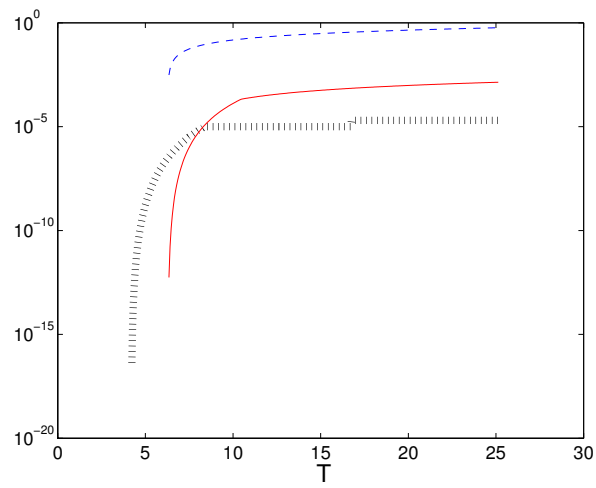

Figure 7: $a: x \mapsto \frac{\beta}{1+4 \pi \beta}\left(1-\frac{\sin (|\omega|)}{|\omega|}\right) \frac{x}{\pi}$ with $\beta=\frac{(\ln 9-\ln 8)^{2}}{2 \pi^{2}}$ and $\omega=(0, \pi / 3)$. Plots of $C_{0}(T,|\omega|)$ $(--), C_{T, T_{0}, 0, \lambda}^{\prime}(/ /)$ and $\widetilde{C}_{T, T_{0}^{\prime}, 0, \lambda}(-)$ with respect to $T$

[4] S. Cox and E. Zuazua, The rate at which energy decays in a damped string, Comm. in partial differential equations 19, (1994), 213-243.

[5] S. Ervedoza and E. Zuazua, On the numerical approximation of controls for waves, Springer Briefs in Mathematics, Springer, New York (2013).

[6] A. Haraux, A generalized internal control for the wave equation in a rectangle, J. Math. Anal. Appl. 153 1990, 190-216.

[7] A Haraux, Séries lacunaires et contrôle semi-interne des vibrations d'une plaque rectangulaire. (French. English summary) [Lacunary series and semi-internal control of the vibrations of a rectangular plate], J. Math. Pures Appl. (9) 68 (1989), no. 4, 457-465 (1990).

[8] A. Haraux, Une remarque sur la stabilisation de certains systèmes du deuxième ordre en temps, Portugaliæ mathematica, 46 (1989), no. 3, 245-258.

[9] A. Henrot, Extremum problems for eigenvalues of elliptic operators, Frontiers in Mathematics, Birkhäuser Verlag, Basel, 2006.

[10] A.E. Ingham, Some trigonometrical inequalities with applications to the theory of series, Math. Zeitschrift 41 (1936), 367-379.

[11] S. Jaffard, S. Micu, Estimates of the constants in generalized Ingham's inequality and applications to the control of the wave equation, Asymptot. Anal. 28 (2001), no. 3-4, 181-214.

[12] S. Jaffard, M. Tucsnak, E. Zuazua, On a theorem of Ingham, J. Fourier Anal. Appl. 3 (1997), $577-582$.

[13] B. Kawohl, Rearrangements and convexity of level sets in PDE, Springer Lecture Notes in Math. 1150, (1985), 1-134.

[14] V. Komornik, P. Loreti Fourier Series in Control Theory, Springer-Verlag, New York, 2005.

[15] V. Komornik, B. Miara Cross-like internal observability of rectangular membranes, Evolution equ. control theory 3 (2014), no.1, 135-146. 
[16] J.-L. Lions, Exact controllability, stabilizability and perturbations for distributed systems, SIAM Rev. 30 (1988), 1-68.

[17] J.-L. Lions, Contrôlabilité exacte, perturbations et stabilisation de systèmes distribués, Tomes $1 \&$ 2, Rech. Math. Appl. [Research in Applied Mathematics], Masson (1988).

[18] F. Periago, Optimal shape and position of the support for the internal exact control of a string, Syst. Cont. Letters 58 (2009), no. 2, 136-140.

[19] J. Pöschel, E. Trubowitz, Inverse spectral theory, Pure and Applied Mathematics, 130. Academic Press, Inc., Boston, MA, 1987. x+192 pp.

[20] Y. Privat, E. Trélat, E. Zuazua, Optimal location of controllers for the one-dimensional wave equation, Ann. Inst. H. Poincaré Anal. Non Linéaire 30 (2013), no. 6, 1097-1126.

[21] Y. Privat, E. Trélat, E. Zuazua, Optimal observation of the one-dimensional wave equation, J. Fourier Anal. Appl. 19 (2013), no. 3, 514-544.

[22] Y. Privat, E. Trélat, E. Zuazua, Optimal observability of the multi-dimensional wave and Schrödinger equations in quantum ergodic domains, Preprint Hal (2012).

[23] D.L. Russell, Controllability and stabilizability theory for linear partial differential equations: recent progress and open questions, SIAM Rev. 20 (1978), no. 4, 639-739.

[24] E. Zuazua, Exact controllability for semilinear wave equations in one space dimension, Ann. Inst. H. Poincaré Anal. Non Linéaire 10 (1993), no. 1, 109-129. 